Review Article

\title{
Memory and Cognition-Related Neuroplasticity Enhancement by Transcranial Direct Current Stimulation in Rodents: A Systematic Review
}

\author{
Carla Cavaleiro, ${ }^{1,2,3}$ João Martins, ${ }^{1,2,3,4}$ Joana Gonçalves $\left(\mathbb{D},{ }^{1,2}\right.$ \\ and Miguel Castelo-Branco ${ }^{1,2,3,4}$ \\ ${ }^{1}$ Coimbra Institute for Biomedical Imaging and Translational Research (CIBIT), University of Coimbra, Coimbra, Portugal \\ ${ }^{2}$ Institute of Nuclear Sciences Applied to Health (ICNAS), University of Coimbra, Coimbra, Portugal \\ ${ }^{3}$ CNC.IBILI Consortium, University of Coimbra, Coimbra, Portugal \\ ${ }^{4}$ Coimbra Institute for Clinical and Biomedical Research (iCBR), Faculty of Medicine, University of Coimbra, Coimbra, Portugal
}

Correspondence should be addressed to Joana Gonçalves; jgoncalves@fmed.uc.pt

and Miguel Castelo-Branco; mcbranco@fmed.uc.pt

Received 6 December 2019; Revised 27 January 2020; Accepted 6 February 2020; Published 26 February 2020

Guest Editor: Luca Marsili

Copyright $\odot 2020$ Carla Cavaleiro et al. This is an open access article distributed under the Creative Commons Attribution License, which permits unrestricted use, distribution, and reproduction in any medium, provided the original work is properly cited.

Brain stimulation techniques, including transcranial direct current stimulation (tDCS), were identified as promising therapeutic tools to modulate synaptic plasticity abnormalities and minimize memory and learning deficits in many neuropsychiatric diseases. Here, we revised the effect of tDCS on the modulation of neuroplasticity and cognition in several animal disease models of brain diseases affecting plasticity and cognition. Studies included in this review were searched following the terms ("transcranial direct current stimulation") AND (mice OR mouse OR animal) and according to the PRISMA statement requirements. Overall, the studies collected suggest that tDCS was able to modulate brain plasticity due to synaptic modifications within the stimulated area. Changes in plasticity-related mechanisms were achieved through induction of long-term potentiation (LTP) and upregulation of neuroplasticity-related proteins, such as $c$-fos, brain-derived neurotrophic factor (BDNF), or N-methylD-aspartate receptors (NMDARs). Taken into account all revised studies, tDCS is a safe, easy, and noninvasive brain stimulation technique, therapeutically reliable, and with promising potential to promote cognitive enhancement and neuroplasticity. Since the use of tDCS has increased as a novel therapeutic approach in humans, animal studies are important to better understand its mechanisms as well as to help improve the stimulation protocols and their potential role in different neuropathologies.

\section{Introduction}

Transcranial direct current stimulation (tDCS) is a noninvasive brain stimulation technique that promotes transient polarity-dependent changes in spontaneous neuronal activity. This effect is mediated by the application of constant low-amplitude electrical currents using epicranially positioned electrodes above a specific brain region of interest [1-4]. The therapeutic use of low-amplitude electrical currents has a long historical track. Accordingly, both Greeks and Romans used electric torpedo fishes for migraine treatment, and in the $11^{\text {th }}$ century, a similar therapeutic procedure was attempted to handle epilepsy [5]. In the $19^{\text {th }}$ century, the application of galvanic currents was attempted to heal melancholia [6]. Over the years, the scientific community interest in brain stimulation grew, and several noninvasive brain stimulation techniques were developed such as tDCS, deep brain stimulation, or transcranial magnetic stimulation. The epicranial application of direct currents promotes a weak electric field force and produces neuronal membrane potential changes $[7,8]$. These alterations occur through sodium and calcium currents [1] modulating spontaneous neuronal activity [2]. The consequent regional neuronal inhibition or excitation depends on the applied current polarity $[9,10]$. So, it was overall observed that cathodal currents produce inhibitory effects, and thus hyperpolarization, 
whereas anodal currents increase excitability in the form of depolarization $[2,11]$ (Figure 1).

There is nowadays an ongoing discussion regarding the factors that interfere with tDCS outcomes. The initial brain resting state of each subject [12], his/her baseline performance [13], specific individual variations in brain tissue morphology [14], or even more particular details from the experimental design or stimulation protocol used [15] influence these outcomes. In vivo and in vitro studies are consensual to demonstrate that tDCS-modulated cortical excitability depends on several stimulation parameters, such as duration and frequency of stimulation [16]; polarity, intensity, and density of the applied current $[17,18]$; and electrode size and position in the scalp [18-20]. Despite that, beneficial effects of tDCS in several brain disorders, such as PD [21, 22], depression [23], stroke [24, 25], or autism [26, 27], have been documented, and there is growing evidence proposing tDCS application in multiple other disease conditions affecting cognition and neuroplasticity mechanisms.

Both preclinical and clinical studies have demonstrated therapeutic effects of tDCS. Indeed, in human studies, anodal tDCS applied intermittently in the prefrontal cortex (PFC) during slow-wave sleep period, improved recall of declarative memories (word pairs). The authors correlated these findings with enhancement of slow oscillatory electroencephalogram (EEG) activity $(<3 \mathrm{~Hz}$, delta $(\delta)$ waves), responsible for neuronal plasticity facilitation [28]. Also, anodal tDCS over dorsolateral prefrontal cortex (DLPFC) improved working memory in PD patients and in major depression patients by boosting cortical excitability [21,23]. Accordingly, preclinical animal studies reported that cortical anodal tDCS improved spatial memory in both wild type (WT) [29] and the $\mathrm{AD}$ rat model [30]. Beneficial effects were also found during the early stage of traumatic brain injury (TBI) [31] and following a pilocarpine-induced status epilepticus in normal rats [32]. Moreover, improvements were also reported concerning short-term memory in an animal model of attention deficit hyperactivity disorder (ADHD) [33].

The molecular mechanisms underlying the tDCSmediated cognitive improvements and neuroplasticity processes have become the focus of recent interest. Accordingly, tDCS modulation over several cognition-related plasticity genes and their signaling pathways has been studied. In this review, we provide a state of the art on the application of different protocols of tDCS in animal models highlighting its effectiveness on neuroplasticity mechanisms and, consequently, their related learning and memory processes. Since the published systematic reviews focused on human application of tDCS, here, we provide a comprehensive revision of the effect of tDCS in in vivo rodent models of normal and pathological brain functioning.

\section{Methods}

2.1. Data Sources and Search. Studies included in this review were identified by searching PubMed. The search was run until 31 October 2019. The search terms were ("transcranial direct current stimulation") AND (mice OR mouse OR animal). Articles were firstly assessed based on their abstracts and titles, aiming to include studies that reported applying tDCS to cognitive impairment in animal models. Simultaneously, the following exclusion criteria were adopted to reject studies: (1) not written in English; (2) performing reviews; (3) in human subjects; (4) in vitro models; (5) employing other brain stimulation techniques (e.g., transcranial magnetic stimulation (TMS), deep brain stimulation (DBS), or transcranial alternating current stimulation (tACS)); and (6) not explicitly describing the tDCS protocol (stimulation area, number of sessions, frequency, intensity, and pattern).

2.2. Data Extraction. A data extraction sheet was developed seeking to retrieve relevant information from each study, namely, study design, sample size, animal model, whether additional therapy was performed, details of the tDCS protocol, outcome measures, and behavioral results.

2.3. Study Selection. The database search was elaborated according to the PRISMA statement requirements [34]. 404 records were found, which underwent a preliminary screening (of titles and abstracts), with 314 records being excluded because they did not meet the eligibility criteria. After the full-text analysis of each of the 90 individual articles, 44 rodent studies focusing on tDCS effects over cognition and neuroplasticity in both healthy and neuropathological animal models were selected (Figure 2).

\section{Results}

3.1. Role of Anodal tDCS in Cognition Processing in Healthy Animals. In healthy animals, studies demonstrated memory improvement in association with induction of synaptic plasticity mechanisms. In fact, tDCS to prefrontal cortex improved monkey's performance on an associative learning task by altering low-frequency oscillations and functional connectivity, both locally and between distant brain areas [35]. Regarding rodent models, data are controversial regarding fear condition. Right frontal anodal tDCS administered $24 \mathrm{~h}$ before behavioral task did not alter contextual and auditory learning and memory [36]. Additionally, another study described that while the anodal stimulation did not affect fear retrieval, posttraining cathodal stimulation improved fear memory retrieval $[37,38]$. However, left prefrontal anodal and cathodal tDCS impaired the acquisition of both contextual and cued fear memory, which could be explained by activity modulation of deep structures such as the amygdala and hippocampus [39].

Concerning learning and memory, de Souza Custódio and colleagues [29] reported better spatial working memory performance following administration of anodal currents to the medial prefrontal cortex (mPFC). In agreement, it was described that administration of hippocampal anodal tDCS improves learning and memory in the Morris water maze and novel object recognition tests [40]. Moreover, memory performance in the passive avoidance learning task was enhanced by anodal stimulation [41]. Also, cortical cathodal stimulation together with visuospatial memory training led to cognitive improvement [42]. 


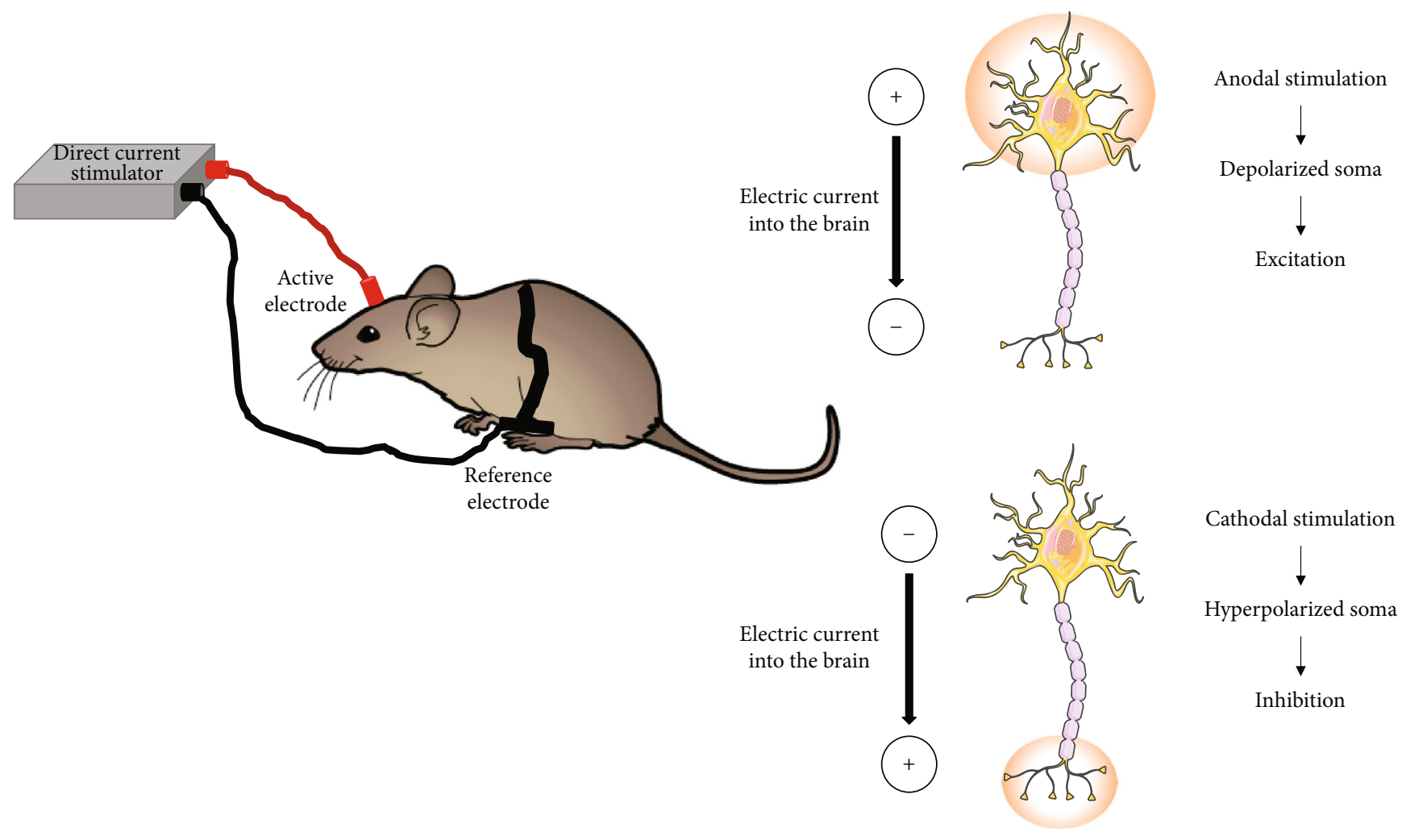

FIGURE 1: Illustration of transcranial direct current stimulation in the mice. Anodal stimulation depolarizes the neuronal membrane and enhances excitability. On the other hand, cathodal stimulation hyperpolarizes the neuronal membrane and decreases excitability.

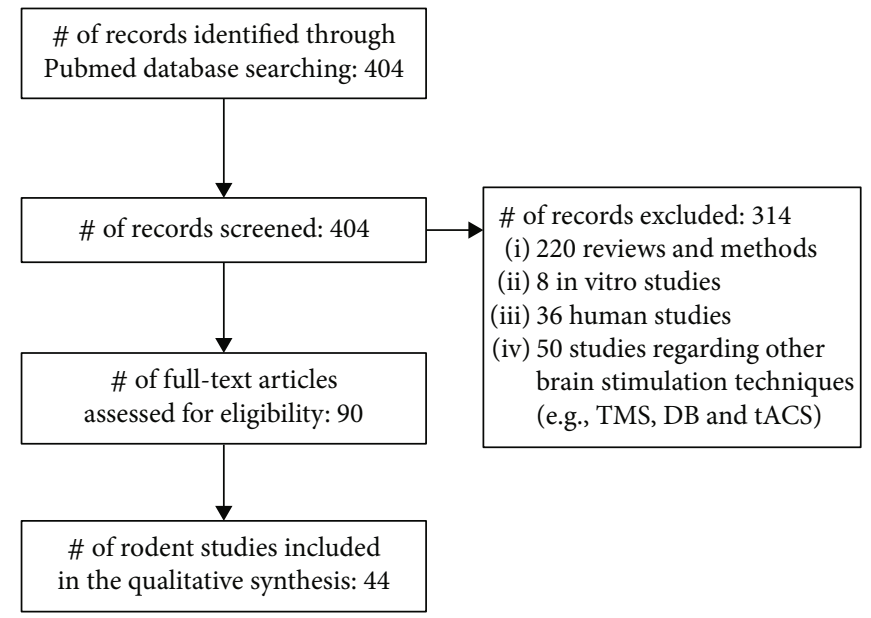

FIGURE 2: Search flow diagram (in accordance with PRISMA statement). Abbreviations: DB: deep brain stimulation; tACS: transcranial alternating current stimulation; TMS: transcranial magnetic stimulation.

The revised in vivo animal model studies regarding tDCS effects in memory and cognition of healthy animals are listed below in Table 1.

3.2. Beneficial Role of tDCS in Brain Diseases. Overall, reports using animal models of brain diseases described a beneficial role of tDCS in the mitigation of memory symptoms of neurologic conditions such as Alzheimer's disease (AD) or traumatic brain injury (TBI). More recent studies demonstrated that tDCS rescued AD-related cognitive symptoms, namely, spatial memory and motor skills $[30,43,44]$. The repetitive stimulation with anodal tDCS in the AD-like dementia rat model reduced the time interval animals needed to reach a food pellet and also decreased the number of errors in the attempt [43]. The same research group showed later that the abovementioned protocol rescued spatial learning and memory in a $\mathrm{A} \beta_{1-40}$-lesioned $\mathrm{AD}$ rat model [30]. Moreover, the impact of tDCS on cognitive performance 


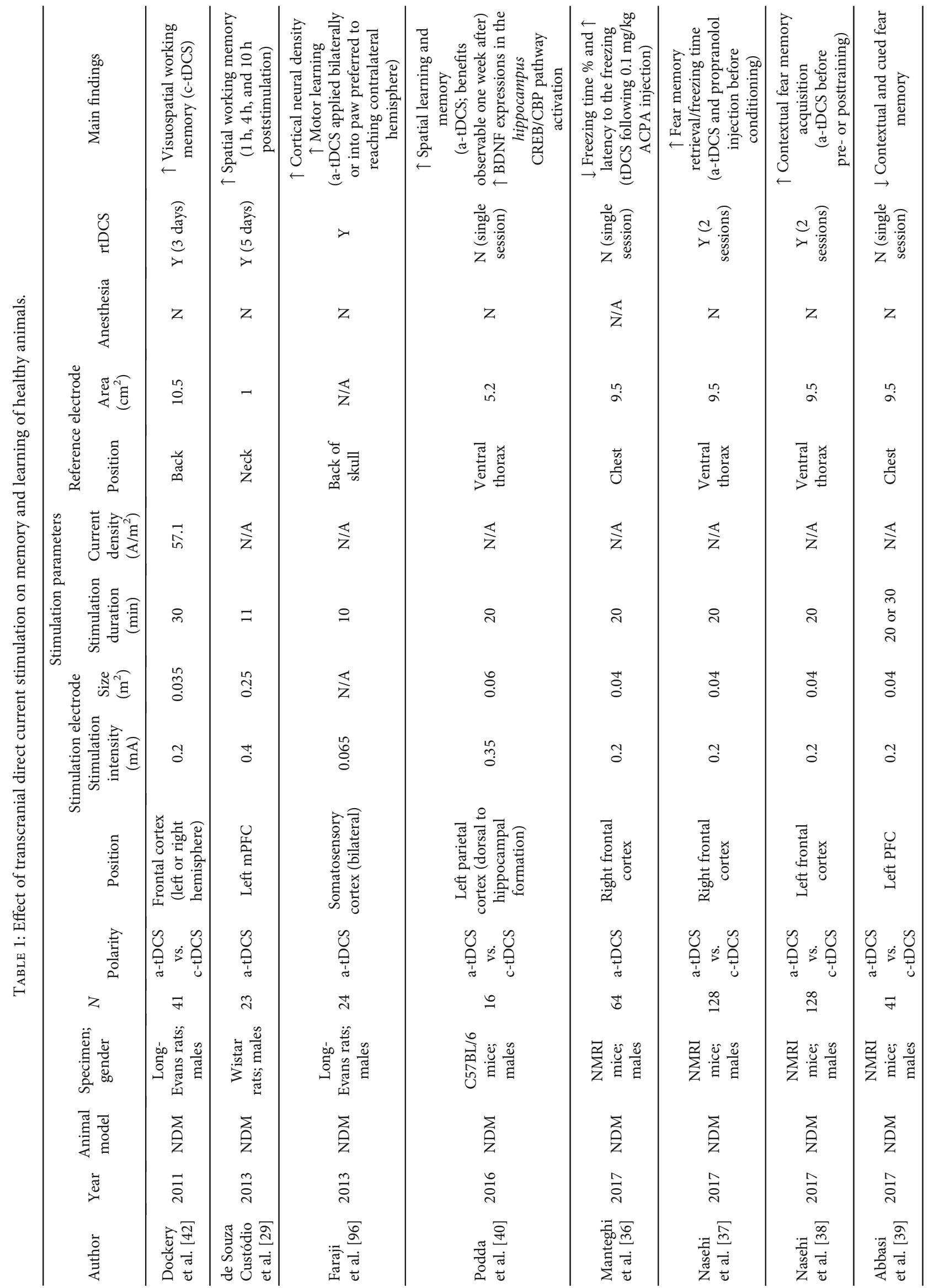




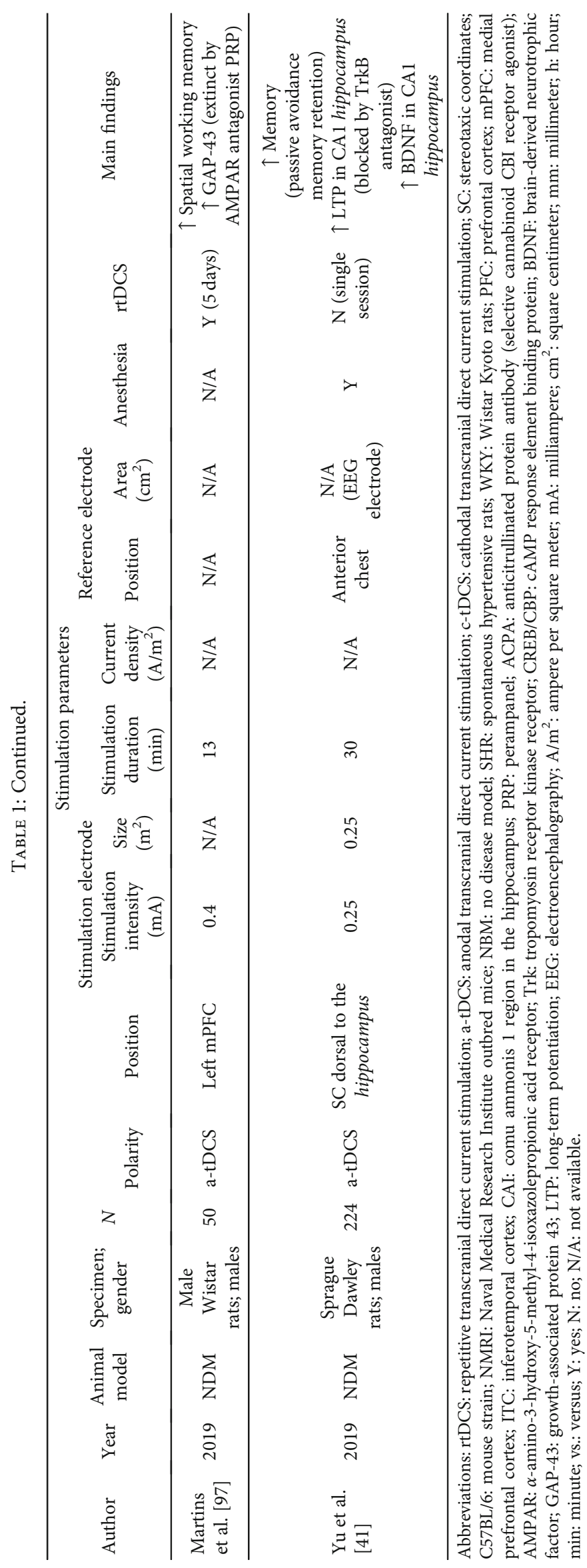


of streptozotocin-induced diabetic rats has been evaluated. Both anodal and cathodal stimulations in the prefrontal cortex restored memory impairment $[45,46]$ together with restoration of LTP [45]. Other authors evaluated the potential therapeutic effects of tDCS in memory impairment in an animal model of ADHD. It was found that this neuromodulation technique was able to improve short- and long-term memory deficits in the spontaneous hypertensive rats (SHR) but not in their control, Wistar Kyoto rats [33, 47]. In addition, no changes were detected in working memory of these control rats following administration of tDCS [47].

Anodal tDCS also ameliorated behavioral and spatial memory function in the early phase after TBI when it was delivered two weeks postinjury. However, earlier stimulation only improved spatial memory [31]. In a later phase of TBI, it was possible to observe motor recovery as well as spatial memory improvement following repeated anodal tDCS [48]. A growing number of studies has been reporting promising effects of neurostimulation in models of addictive disorders, by reducing craving and maladaptive pervasive learning [49]. In fact, repeated anodal stimulation in mouse frontal cortex decreased nicotine-induced conditioned place preference and further improved working memory [50]. Same polarity currents also could prevent cocaine-induced locomotor hyperactivity and place preference conditioning [51]. In addition, it has been reported that cathodal stimulation has an anticonvulsive effect [16, 32, 52-54]. Indeed, the administration of hippocampal tDCS rescued cognitive impairment by reducing hippocampal neural death and supragranular and CA3 mossy fiber sprouting in a lithiumpilocarpine-induced status epilepticus rat [32]. Other neuroplastic effects were evidenced in the reversion of motor symptoms in PD by tDCS administration. The application of anodal currents enhanced graft survival and dopaminergic re-innervation of the surrounding striatal tissue and pronounced behavioral recovery [55].

Despite the fact that many studies reported recovery from memory deficits following tDCS stimulation, there are some opposing reports in animal models of disease affecting cognition. In a recent study from Gondard and collaborators using a triple transgenic ( $3 \times \mathrm{Tg}$ ) mouse model of $\mathrm{AD}$, it was evidenced that a neurostimulation was not able to ameliorate memory symptoms [56]. To reconcile this discrepancy, previous authors have suggested the importance of choosing an optimal current intensity in order to modulate cortical excitability since LTP alterations were dependent on current intensity [57].

The reports regarding tDCS effects in cognition and memory in animal models of brain disease are listed in Tables 2 and 3.

\subsection{Effect of tDCS on Cellular and Molecular Neuroplasticity} Mechanisms. Neuronal network reorganization underlies neuroplasticity processes like developmental synaptogenesis, or neurogenesis and synaptic turnover later on, which ultimately contributes to optimal brain development and aging, as well as functional recovery upon trauma [58]. Interestingly, several reports using genetic engineered animals, phar- macologically induced animal models of disease, or in vitro techniques enlightened the potential of direct current stimulation (DCS) to interact with a myriad of neuroplasticityrelated processes such as neuroinflammation $[59,60]$, neural stem cell migration [59], neurite growth [61], or neurogenesis [62]. Moreover, both human and in vivo animal studies evidenced a tDCS-induced effect on memory and learning $[28,35,63]$. However, the underlying cellular and molecular mechanisms remain to be elucidated.

3.3.1. Modulation of the Excitatory/Inhibitory Network. To date, animal experimental evidence highlighted tDCS influences on synaptic plasticity, through alterations in the functional connectivity of cognition-related areas [35] and by modulation of excitatory/inhibitory network tonus [64], which may involve both the GABAergic and glutamatergic systems. Accordingly, a study conducted with older adults remarked an anodal stimulation effect in gamma-aminobutyric acid (GABA) levels [65]. Similarly, in human healthy volunteers, an anodal tDCS effect in motor learning was correlated with a decrease in GABA levels, an outcome known to be a determinant factor in the promotion of long-term potentiation- (LTP-) dependent plasticity and therefore learning $[66,67]$.

Several preclinical studies probed LTP enhancement following direct current stimulation. Anodal DCS enhanced LTP in both mouse cortex [68] and rat hippocampal slices $[69,70]$. Further, this neurostimulation method increased local field potential (LFPs) amplitudes in primary somatosensory cortex of rabbits [63]. Also, other works demonstrated that neurostimulation-enhanced hippocampal LTP was associated with better spatial memory performance along with an increase in brain-derived neurotrophic factor (BDNF) expression levels [40]. An opposite effect on LTP and LFPs was obtained with administration of cathodal currents. In agreement, a report from Sun et al. [71] evidenced that cathodal currents applied in mouse neocortical slices induced field excitatory postsynaptic potential depression. This type of LTD was smothered by application of an mGluR5 negative allosteric modulator [72]. These findings support a possible modulatory effect of tDCS on mGluR5mTOR signaling [72]; these molecular pathways are recognized to disturb cognition-related synaptic plasticity.

Further evidence supporting tDCS effect on LTP-like mechanisms was recently brought to light by Stafford et al. [73]. These authors observed that a single anodal tDCS increased both the phosphorylation at the S831 of GluA1 subunit and the translocation of $\alpha$-amino-3-hydroxy5methyl-4-isoxazole propionic acid receptors (AMPARs) from cytosolic to synaptic fractions in the hippocampus. These data could be favoring learning enhancement, as this translocation has been associated with hippocampal LTP induction [72]. Accordingly, others reported a spatial working memory enhancement after anodal stimulation over left medial PFC that was lost with the administration of the AMPAR antagonist perampanel (PRP). In contrast to cathodal currents, anodal currents enhanced intracellular calcium $\left(\mathrm{Ca}^{2+}\right)$ intake in cell cultures including astrocytes [74-76], a process associated with AMPAR phosphorylation 


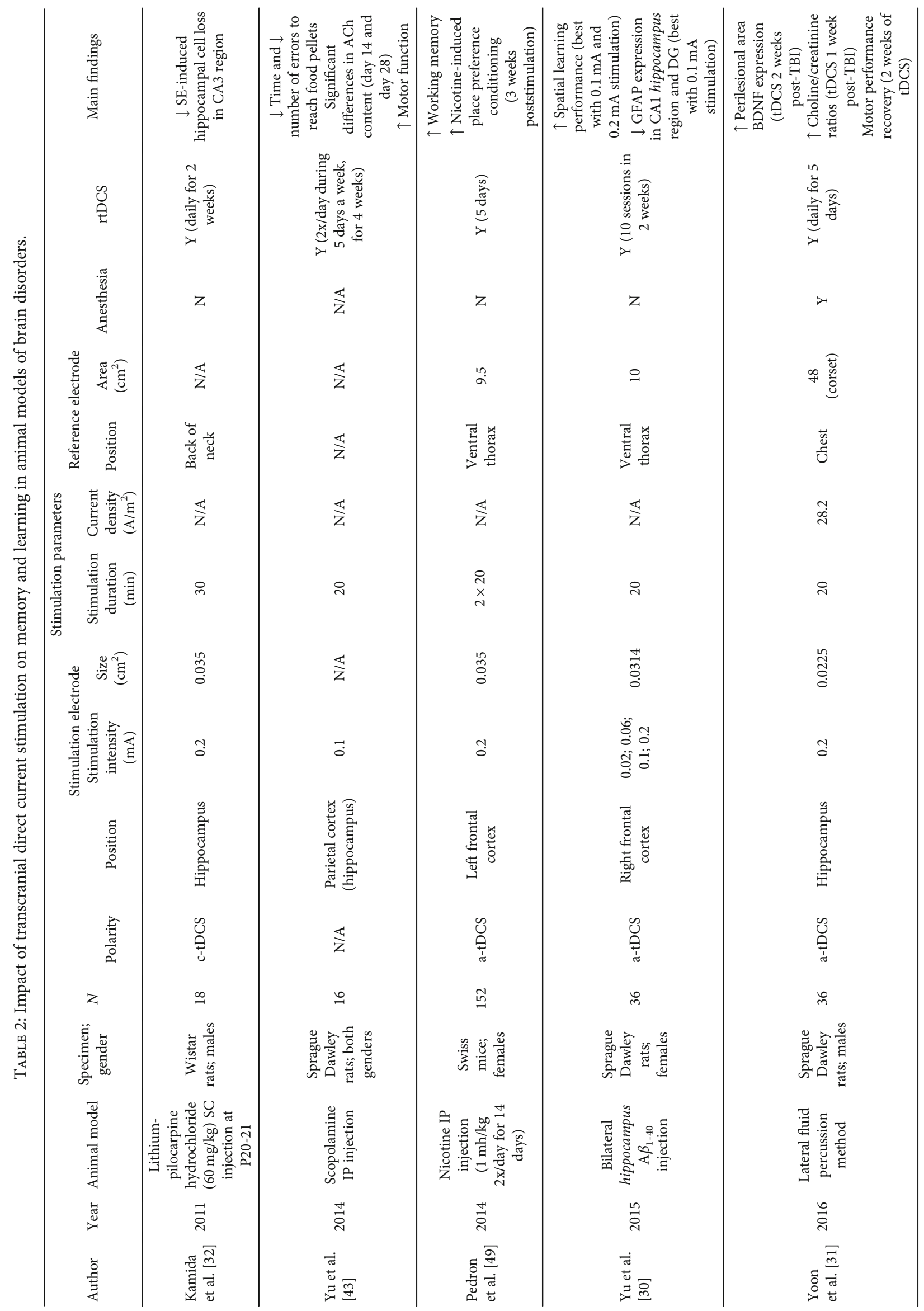




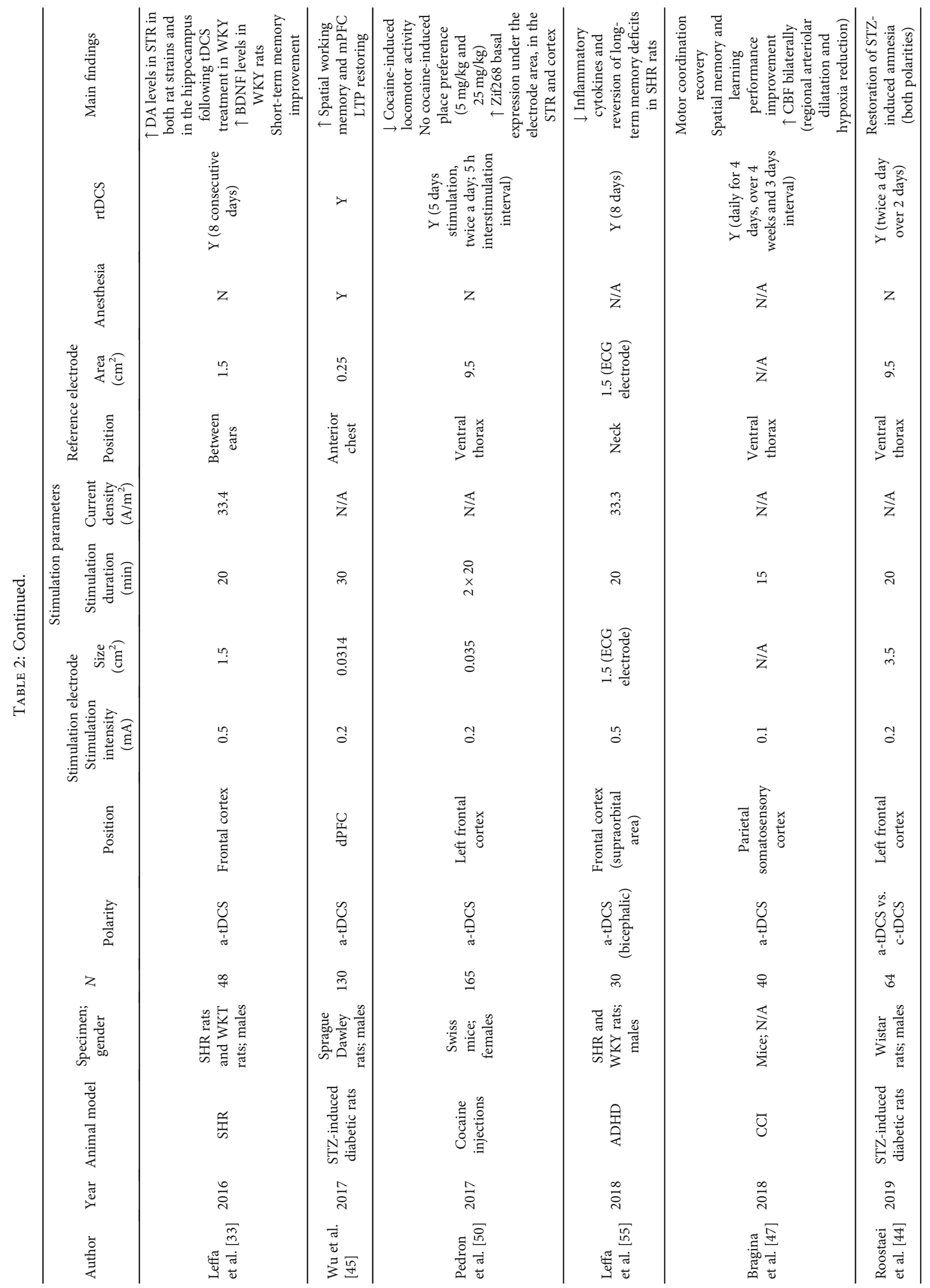




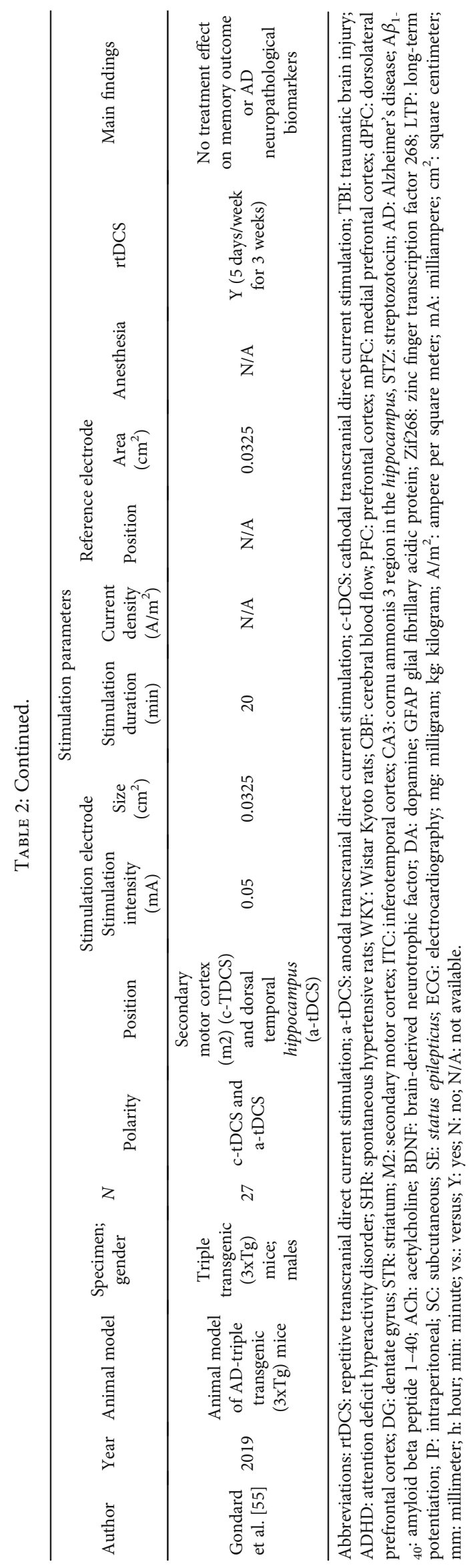




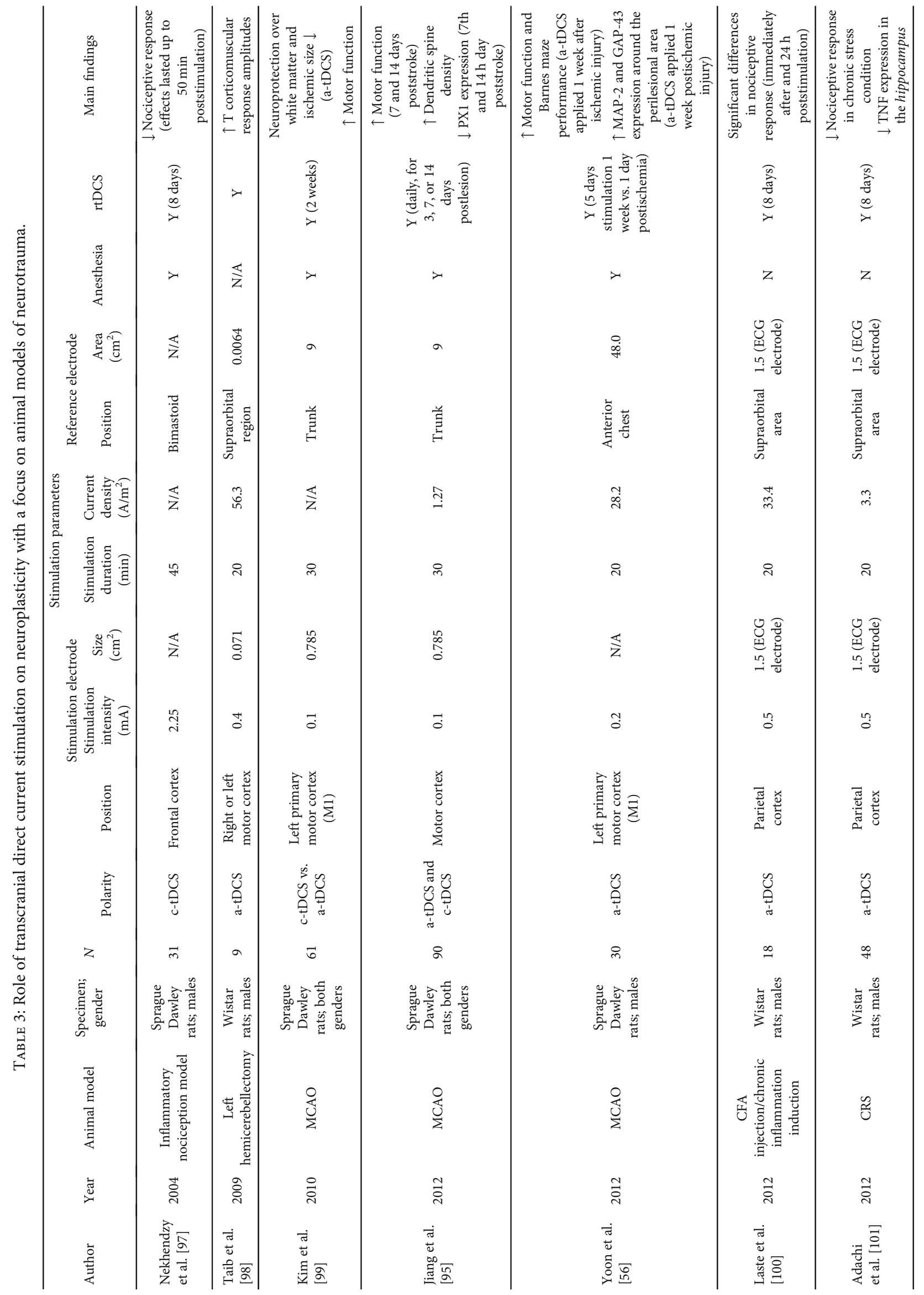




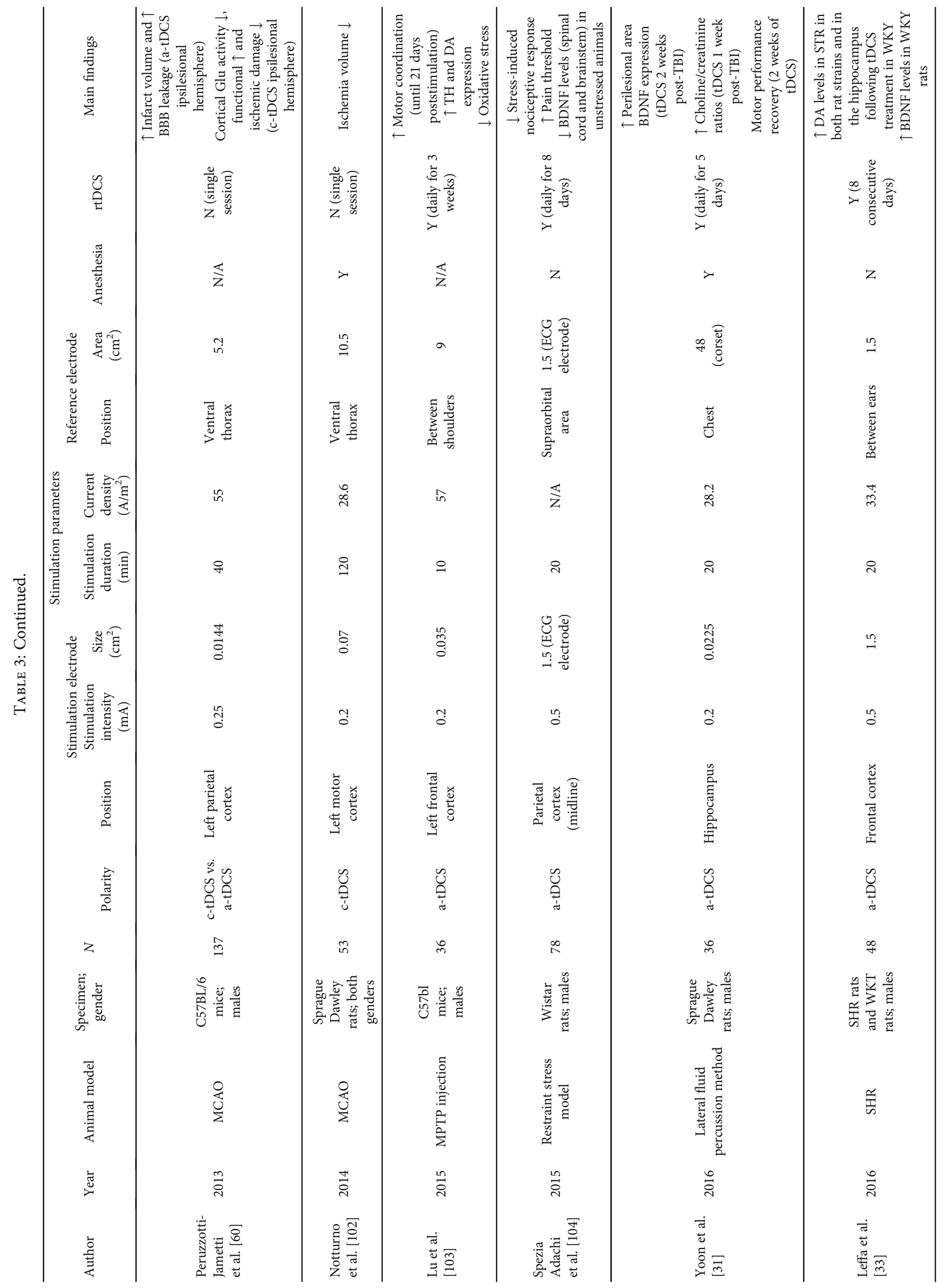




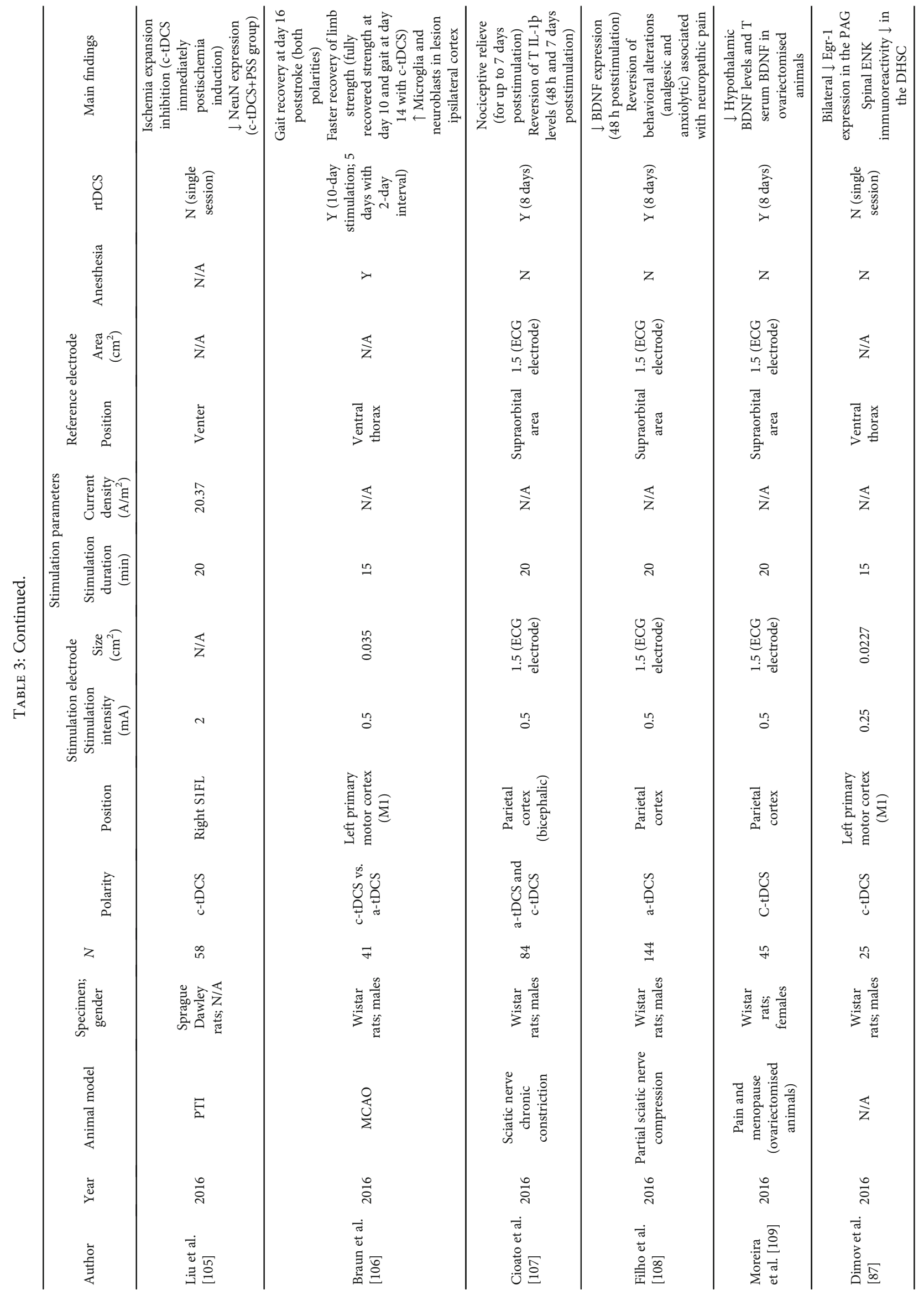




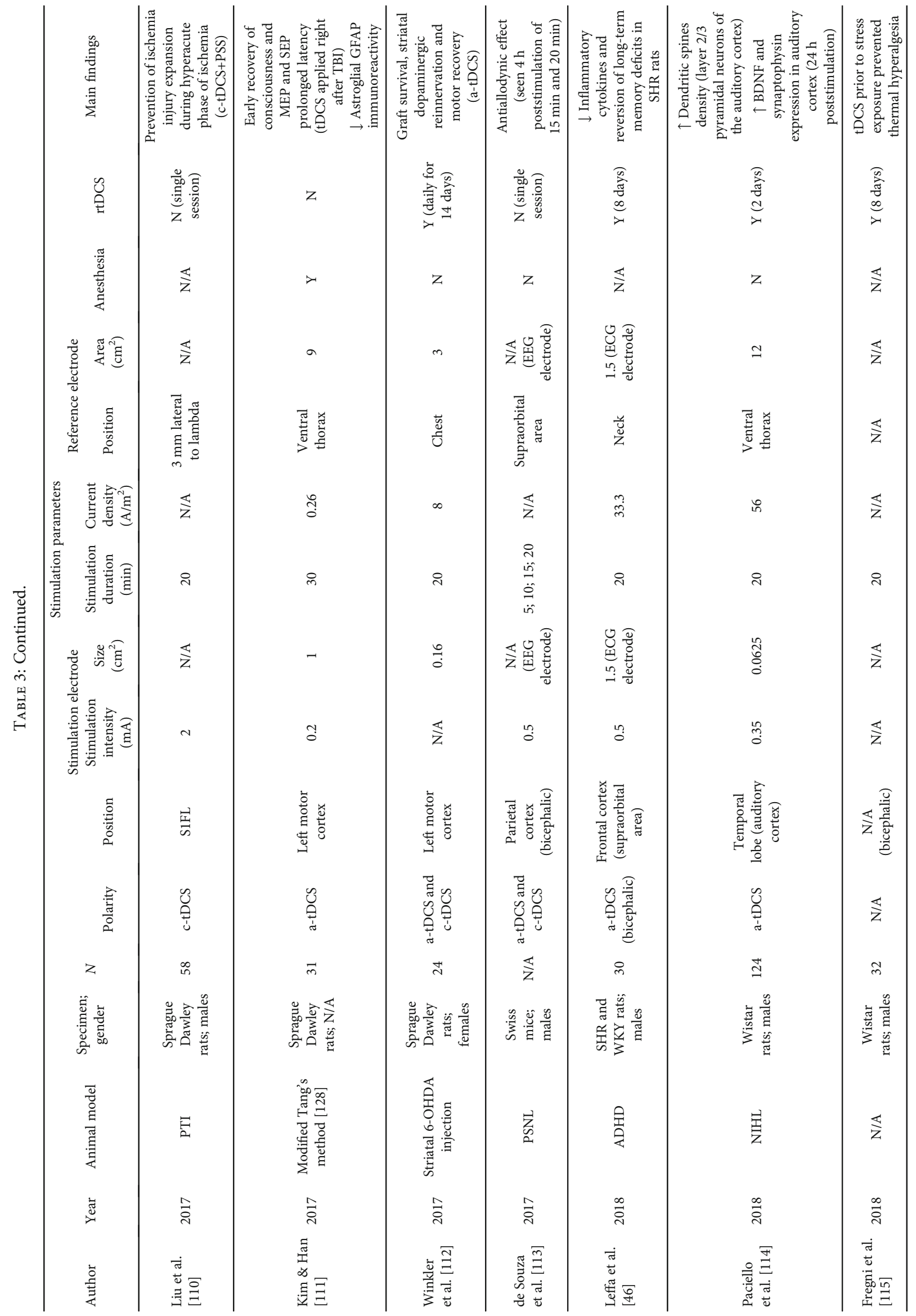




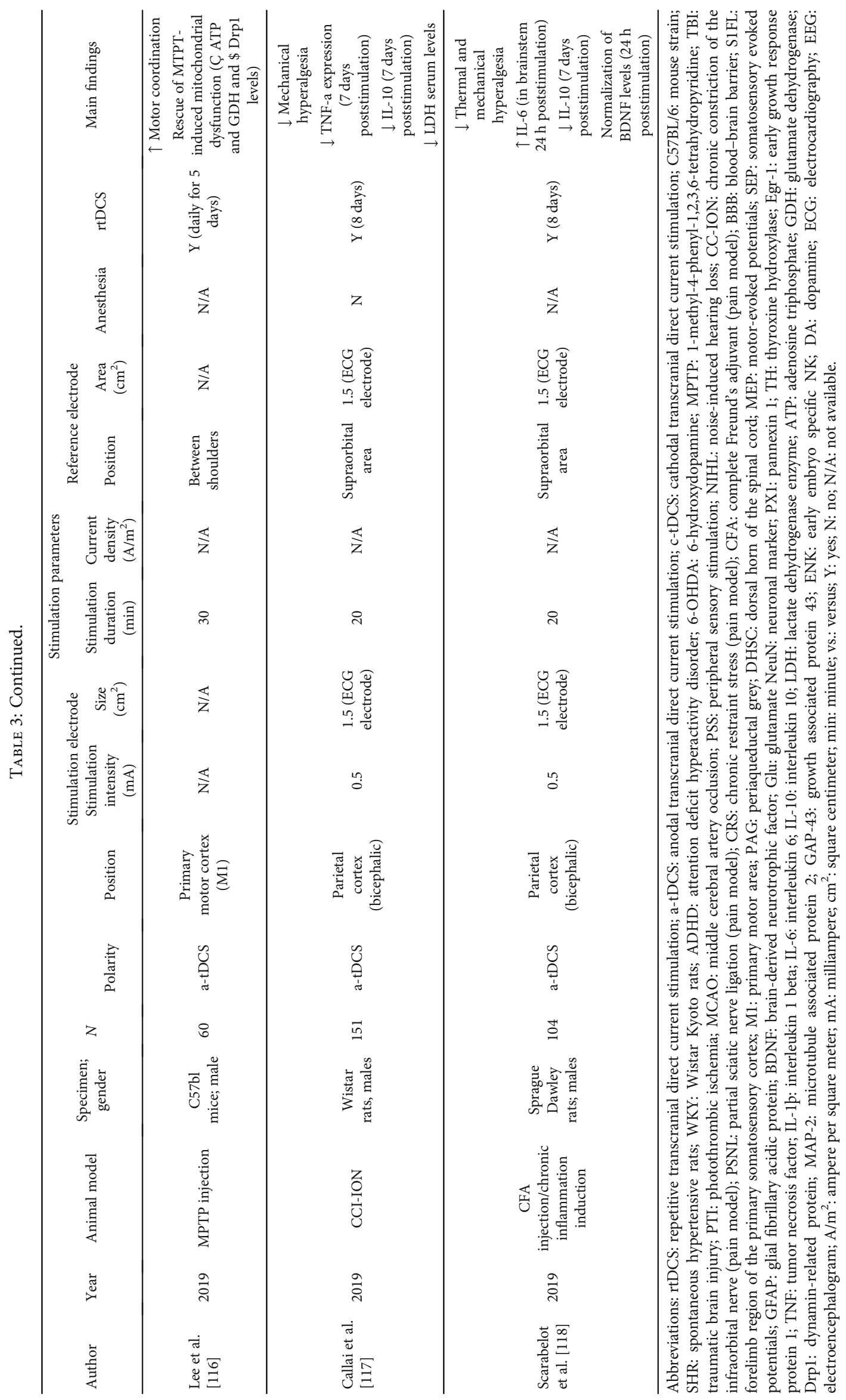


nd trafficking to postsynaptic density [77] and ultimately, allowing LTP facilitation, a cellular correlate of learning and memory.

3.3.2. Activation of Neuroplasticity-Associated Gene Expression. Neurostimulation could have long-lasting effects in memory as data from different studies evidenced [40]. Authors have been argued that tDCS cognition modulation is associated with neuroplasticity-associated gene expression alterations [78]. One of the neuroplasticity-associated genes, known to be essential for hippocampal LTP, is BDNF [79]. Several studies elucidated the role of BDNF in memory modulation by tDCS. In fact, it was reported that anodal currents could increase BDNF expression [68], and its activation via tropomyosin receptor kinase (Trk) receptors [80], triggering NMDAR opening, and inducing a later phase LTP (L-LTP) facilitation [81]. Accordingly, Yu et al. [41] found that the administration the Trk inhibitor ANA-12 prevented the anodal tDCS-induced hippocampal CA1 LTP increase. Other studies, using the same polarity currents, revealed a link between the upregulation of BDNF and cAMP response element binding protein/CREB-binding protein (CREB/CBP) [40] involved in LTP and memory formation [82]. Also, the application of cortical anodal currents in frontal cortex was able to upregulate BDNF together with striatal dopamine [33]. The upregulation of BDNF following neurostimulation was associated with the augmentation of expression levels of immediate early genes (IEGs), such as c-fos and zif268 [69]. Moreover, Kim et al. [78] confirmed that repetitive anodal tDCS in right sensorimotor cortex of healthy rats promoted a significant increase of mRNA levels of plasticityassociated genes, namely, BDNF, cAMP response element binding protein (CREB), synapsin I, $\mathrm{Ca}^{2+} /$ calmodulindependent protein kinase II (CaMKII), activity-regulated cytoskeleton-associated protein (Arc), and c-fos. It was also demonstrated that sensory evoked cortical responses were boosted after tDCS via alpha-1 adrenergic receptormediated astrocytic $\mathrm{Ca}^{2+} / \mathrm{IP} 3$ signaling, thus involving also glia and the adrenergic system [75]. Anodal tDCS actions in glia were further confirmed by Mishima et al. [76]. Using a mouse model lacking $\mathrm{Ca}^{2+}$ uptake in astrocytes, the inositol trisphosphate receptor type 2 (IP3R2) knockout (KO) mouse and also an adrenergic receptor antagonist, they confirmed decreased microglia motility along with soma enlargement in tDCS stimulated animals [76].

In poststroke recovery, it was found that anodal currents significantly increased the GAP-43 and the microtubuleassociated protein 2 (MAP-2) expression around the infarct area [56]. These neuronal growth-promoting proteins are overexpressed during dendritic remodeling and axonal regrowth throughout the acute phase of stroke $[83,84]$. Anodal stimulation also modulated pannexin-1 (PX1) hemichannel levels $[85,86]$ and, following an ischemic insult, neurostimulation decreased rat PX1 mRNA and, consequently, augmented dendritic spine density in the surrounding areas of cerebral infarction; these cellular outcomes were associated with the improvement of motor function [85]. Some authors proposed that tDCS-induced improvement of stroke/TBI symptoms might be due to increase of BDNF expres- sion and associated with choline/creatine ratios in the perilesional cortex [31].

Overall, tDCS methodology was able to modulate molecular pathways involved in the regulation of cognition-related synaptic plasticity mechanisms (Figure 3 ). The revised in vivo animal studies regarding tDCS-induced effects in the cellular and molecular mechanisms of memory and learning are listed in Table 4.

\section{Discussion}

This systematic review collected several studies that confirm the potential effects of tDCS on neuronal activity and synaptic plasticity. Here, we documented a variable combination of stimulation protocols, stimulation areas, and healthy and disease animal models. Most of the existent literature is focused on human application of tDCS. The comprehensive revision of the effect of tDCS on rodent models of normal and pathological brain functioning does therefore provide a novel contribution to the field. Overall, the revised studies indicated that tDCS was able to modulate synaptic plasticity and, consequently, learning and memory processes $[87,88]$.

Memory formation and consolidation are recognized to rely on activity-dependent modifications, such as LTD and LTP [89], both dependent on the activation of calciumdependent kinases (e.g., CaMKs), which in turn control the trafficking of NMDARs and AMPARs [90]. Despite the wide set of stimulation protocols, tDCS-induced modulation of NMDAR signaling and synaptic protein upregulation resulting in LTP and cognitive enhancement have been consistently reported in animal studies. Anodal tDCS increased AMPAR synapse translocation $[73,89]$ and induced spatial memory improvement by involving both CREB and BDNF expression alterations [53]. Also, an increase in hippocampal and cortical mRNA levels of c-fos, synapsin, CaMKII, and Arc was observed poststimulation [78].

Similar results highlighting tDCS effects in neuroplasticity were obtained with in vitro studies. Accordingly, Ranieri and coworkers [69] probed that anodal currents increased NMDAR-dependent LTP in hippocampal CA3-CA1 synapses [69], in part, due to production of BDNF [68]. In addition, it was demonstrated that tDCS-induced hippocampal BDNF release is dependent on histone acetylation of BDNF gene promoters [40]. Overall, the abovementioned works provide positive evidence for the effect of tDCS on cognitive function enhancement.

Although tDCS impaired the acquisition of both contextual and cued fear memory [39], there are no studies on possible cascades/proteins involved in tDCS-induced neuroplasticity alterations following fear memory changes. Nevertheless, a very recent paper demonstrated chronic repetitive TMS of the ventromedial prefrontal cortex reversed stress-induced behavior impairments with an increase of c-fos activity [91].

Cortical anodal currents have been shown to be mostly excitatory and support memory enhancement and neuroplasticity. The literature is also consistent with the notion that the stimulation over the cortical region functionally involved in a certain cognitive task increases performance in that specific task. Marshall et al. demonstrated that anodal 


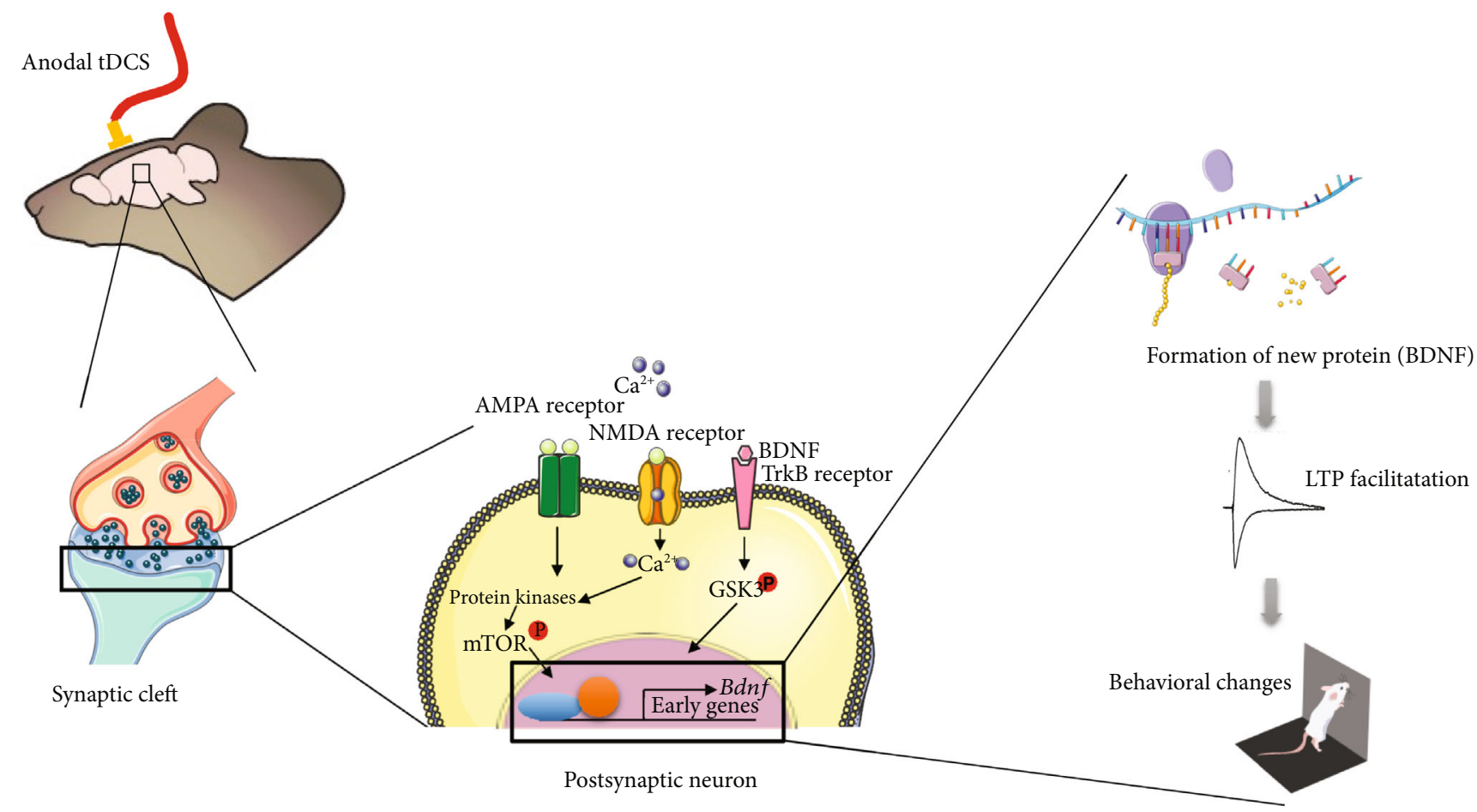

FIGURE 3: Schematic illustration of molecular mechanisms underlying the effect of anodal transcranial direct current stimulation (tDCS) on neuronal physiology. The neurostimulation in the target cortical area depolarizes neuronal membrane and glutamate released in presynaptic neuron and binds in NMDA and AMPA receptors (see book chapter Rozisky et al., 2015). Consequently, there is intracellular Ca ${ }^{2+}$ upregulation in the postsynaptic neuron, which can activate protein kinases that in turn modulate numerous neuronal signaling pathways (such as the mTOR pathway) leading to transcriptional changes. The tDCS also activates molecular cascades to promote BDNF production. As a long-term mechanism, gene transcription is modulated leading to the formation of new proteins that in turn lead to facilitation of LTP and improvement of cognition. Abbreviations: AMPA: $\alpha$-amino-3-hydroxy-5-methyl-4-isoxazolepropionic acid; BDNF: brain-derived neurotrophic factor; CBP: CREB-binding protein; CREB: cAMP response element binding protein; GSK3: glycogen synthase kinase 3; LTP: long-term potentiation; mTOR: mammalian target of rapamycin; NMDA: N-methyl-D-aspartate; TrkB: tropomyosin receptor kinase $\mathrm{B}$.

currents over the PFC, a region involved in memory encoding, during slow wave sleep improved declarative memory [28]. However, it was described that cortical cathodal stimulation simultaneously with training task was able to increase visuospatial working memory, in spite of the fact that it was associated with decreased excitability [42]. This suggests that modulatory effects of tDCS were influenced by the polaritydependent electrical dynamics established between the stimulated area and its related neuronal networks. In agreement, a recent report observed an inhibitory effect in motor learning tasks following anodal currents in the cerebellum; the anodal excitatory effect over the Purkinje cell activity led to an overall inhibition of downstream structures, reducing as a result the vestibulo-ocular reflex gain [90]. Similar paradoxical results have been observed in humans. Recently, Moliadze and collaborators [92] reported that tDCSinduced neural modulation depended on several parameters, namely, the age. In fact, an excitatory effect was seen in young subjects, but not in the older participants.

Nowadays, TMS, another important noninvasive brain stimulation technique, is useful for evaluating excitability in the primary motor cortex (M1) and conductivity along the cortical-spinal tract. This technique has been amply used in rehabilitation of stroke patients [93] and in neuropsychiatric disorders, namely, depression [94]. tDCS and TMS are undergoing the most active investigation and share a capacity to modulate regional cortical excitability, and both are welltolerated by children and adults [95]. However, TMS has been already approved for clinical use and tDCS is still undergoing investigation as a plausible therapy for a range of neuropsychiatric disorders [95]. The rational, in part, for this is because data on the efficacy and safety of tDCS are sparse and employ heterogeneous stimulation protocols. Indeed, there is a paucity of strictly conducted randomized, sham controlled clinical trials, and case considerable followup periods, which makes it difficult to use these results to inform clinical practice concerning the putative beneficial role of tDCS. Moreover, tDCS effects seem to be clearly dependent on structure, connectivity, and function of the target brain region. Importantly, these outcomes were intrinsically correlated with GABAergic neurotransmission which raises the issue that one has to take into account that during development GABA can act as an excitatory neurotransmitter [96].

\section{Conclusions}

There is growing evidence that tDCS modulates brain activity and, consequently, enhances synaptic plasticity and cognitive 


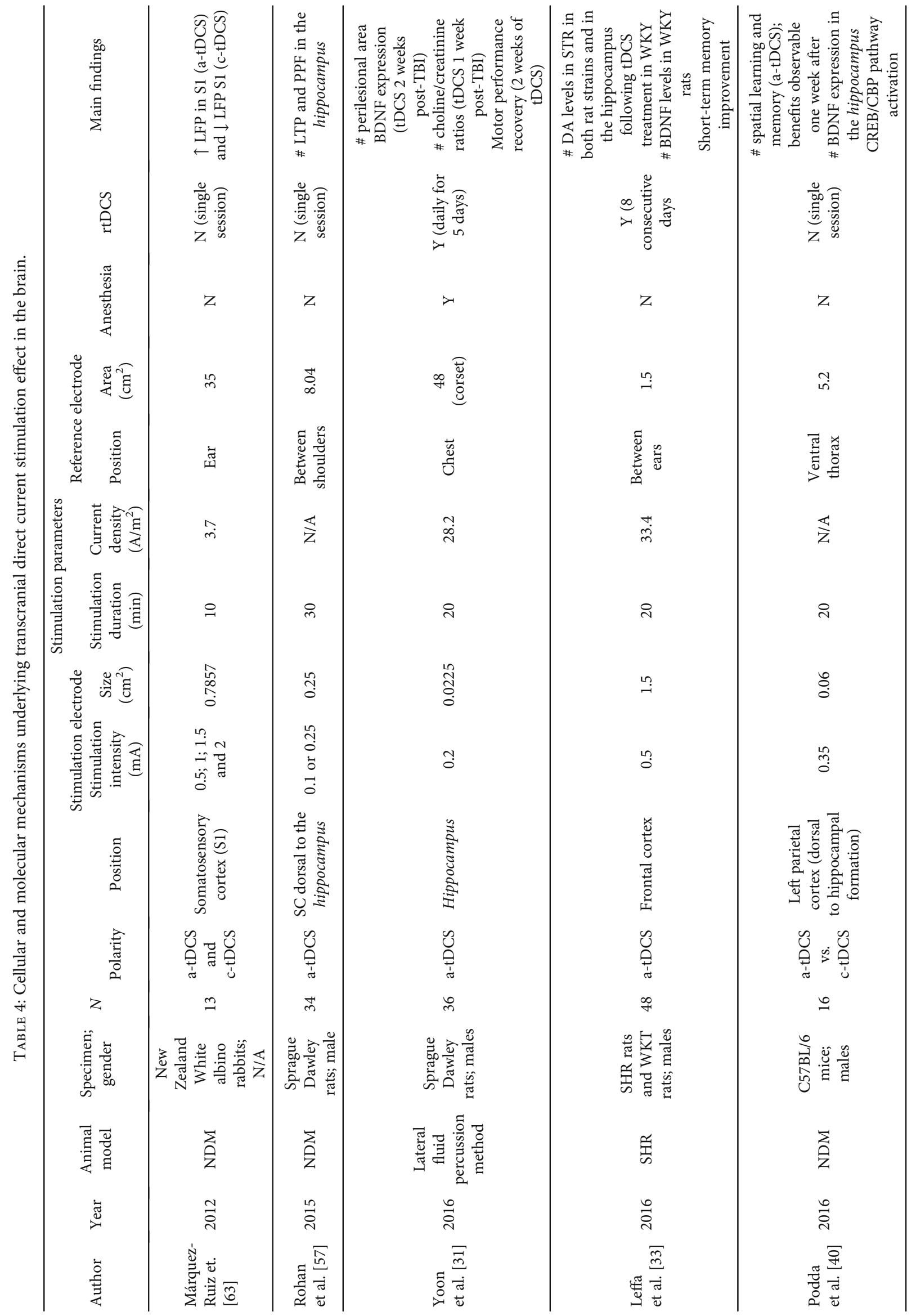




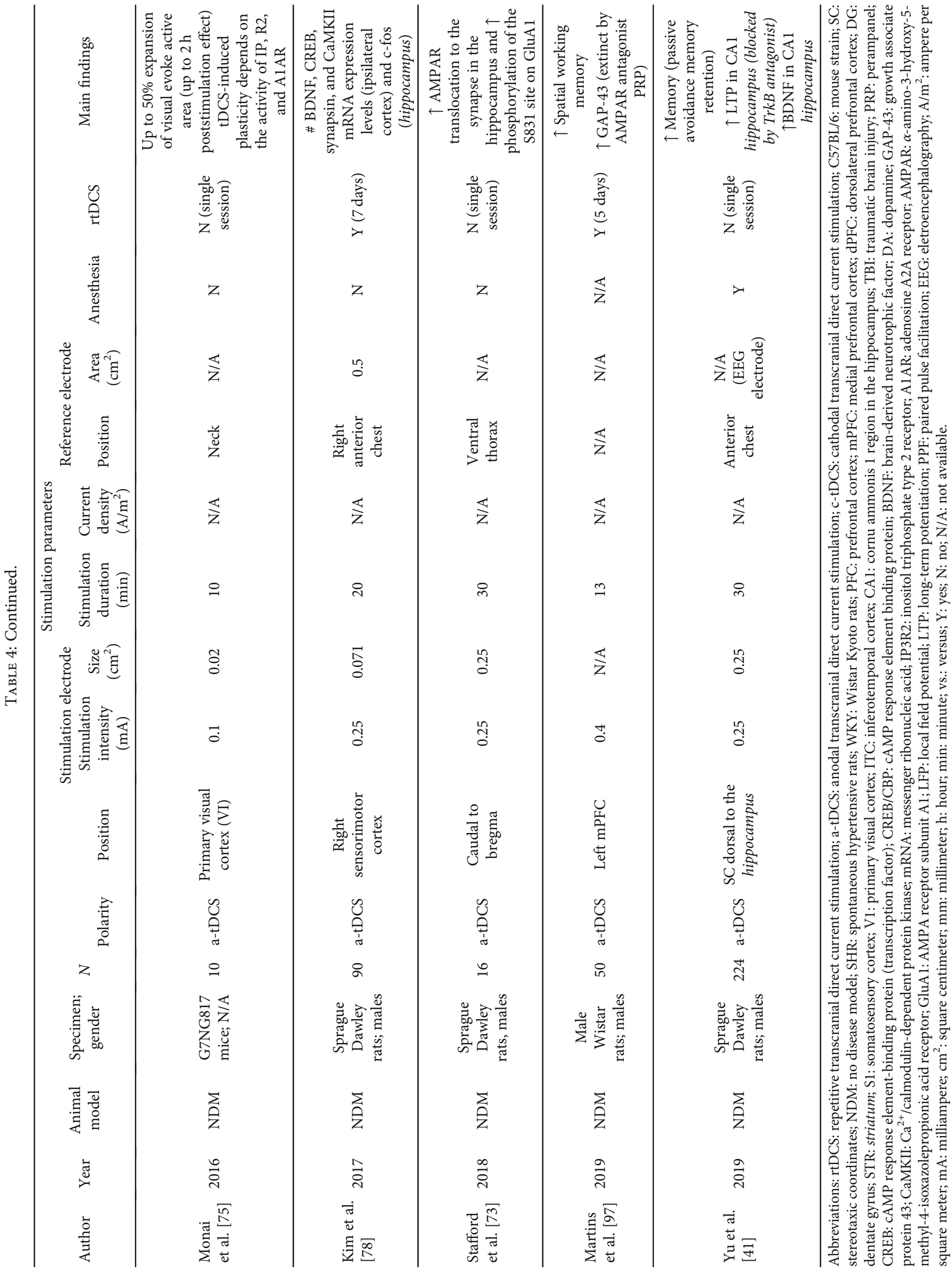


performance. Overall, reports from laboratory animal research present tDCS as a promising noninvasive brain stimulation technique. The presented evidence is therefore consistent with human studies suggesting that this technique is useful to mitigate neurologic symptoms of several brain disorders, thus improving learning and memory. Further research is needed so that this technique can be fully translated into optimal therapeutic strategies.

\section{Conflicts of Interest}

The authors declare that there is no conflict of interest regarding the publication of this paper.

\section{Authors' Contributions}

Joana Gonçalves and Miguel Castelo-Branco share senior authorship.

\section{Acknowledgments}

This work was supported by grants POCI-01-0145-FEDER016428 and CENTRO-01-0145-FEDER-000016 financed by Centro 2020 FEDER, COMPETE, FLAD Life Sciences Ed 2 2016, FCT/UID 4950 COMPETE, POCI-01-0145-FEDER007440, FCT, and European Grant H2020 STIPED.

\section{References}

[1] G. Ruffini, F. Wendling, I. Merlet et al., "Transcranial current brain stimulation (tCS): models and technologies," IEEE Transactions on Neural Systems and Rehabilitation Engineering, vol. 21, no. 3, pp. 333-345, 2013.

[2] M. A. Nitsche and W. Paulus, "Excitability changes induced in the human motor cortex by weak transcranial direct current stimulation," The Journal of Physiology, vol. 527, no. 3, pp. 633-639, 2000.

[3] T. Wagner, A. Valero-Cabre, and A. Pascual-Leone, "Noninvasive human brain stimulation," Annual Review of Biomedical Engineering, vol. 9, no. 1, pp. 527-565, 2007.

[4] M. Nitsche, D. Liebetanz, A. Antal, N. Lang, F. Tergau, and W. Paulus, "Modulation of cortical excitability by weak direct current stimulation-technical, safety and functional aspects," Supplements to Clinical Neurophysiology, vol. 56, pp. 255276, 2003.

[5] P. Kellaway, "The part played by electrical fish in the early history of bioelectricity and electrotherapy," Bulletin of the History of Medicine, vol. 20, no. 2, pp. 130-134, 1946.

[6] A. Parent, "Giovanni Aldini: from animal electricity to human brain stimulation," The Canadian Journal of Neurological Sciences, vol. 31, no. 4, pp. 576-584, 2004.

[7] T. Bullock and C. Terzuolo, "Diverse forms of activity in the somata of spontaneous and integrating ganglion cells," The Journal of Physiology, vol. 138, no. 3, pp. 341-364, 1957.

[8] C. Poreisz, K. Boros, A. Antal, and W. Paulus, "Safety aspects of transcranial direct current stimulation concerning healthy subjects and patients," Brain Research Bulletin, vol. 72, no. 46, pp. 208-214, 2007.

[9] L. Bindman, O. Lippold, and J. Redfearn, "The action of brief polarizing currents on the cerebral cortex of the rat (1) during current flow and (2) in the production of long-lasting after- effects," The Journal of Physiology, vol. 172, pp. 369-382, 1964.

[10] C. A. Terzuolo and T. H. Bullock, "Measurement of imposed voltage gradient adequate to modulate neuronal firing," Proceedings of the National Academy of Sciences of the United States of America, vol. 42, no. 9, pp. 687-694, 1956.

[11] M. A. Nitsche and W. Paulus, "Sustained excitability elevations induced by transcranial DC motor cortex stimulation in humans," Neurology, vol. 57, no. 10, pp. 1899-1901, 2001.

[12] A. Antal, D. Terney, C. Poreisz, and W. Paulus, "Towards unravelling task-related modulations of neuroplastic changes induced in the human motor cortex," The European Journal of Neuroscience, vol. 26, no. 9, pp. 2687-2691, 2007.

[13] B. Krause and R. Cohen Kadosh, "Not all brains are created equal: the relevance of individual differences in responsiveness to transcranial electrical stimulation," Frontiers in Systems Neuroscience, vol. 8, 2014.

[14] M. Bikson, A. Rahman, and A. Datta, "Computational models of transcranial direct current stimulation," Clinical EEG and Neuroscience, vol. 43, no. 3, pp. 176-183, 2012.

[15] A. Fertonani and C. Miniussi, "Transcranial electrical stimulation: what we know and do not know about mechanisms," The Neuroscientist, vol. 23, no. 2, pp. 109-123, 2017.

[16] D. Liebetanz, F. Klinker, D. Hering et al., "Anticonvulsant effects of transcranial direct-current stimulation (tDCS) in the rat cortical ramp model of focal epilepsy," Epilepsia, vol. 47, no. 7, pp. 1216-1224, 2006.

[17] D. Liebetanz, R. Koch, S. Mayenfels, F. König, W. Paulus, and M. A. Nitsche, "Safety limits of cathodal transcranial direct current stimulation in rats," Clinical Neurophysiology, vol. 120, no. 6, pp. 1161-1167, 2009.

[18] M. Jackson, D. Truong, M. Brownlow et al., "Safety parameter considerations of anodal transcranial direct current stimulation in rats," Brain, Behavior, and Immunity, vol. 64, pp. 152-161, 2017.

[19] M. Nitsche, L. Cohen, E. Wassermann et al., "Transcranial direct current stimulation: state of the art 2008," Brain Stimulation, vol. 1, no. 3, pp. 206-223, 2008.

[20] K. Monte-Silva, M. Kuo, D. Liebetanz, W. Paulus, and M. A. Nitsche, "Shaping the optimal repetition interval for cathodal transcranial direct current stimulation (tDCS)," Journal of Neurophysiology, vol. 103, no. 4, pp. 1735-1740, 2010.

[21] P. S. Boggio, R. Ferrucci, S. P. Rigonatti et al., "Effects of transcranial direct current stimulation on working memory in patients with Parkinson's disease," Journal of the Neurological Sciences, vol. 249, no. 1, pp. 31-38, 2006.

[22] M. Horiba, Y. Ueki, I. Nojima et al., "Impaired motor skill acquisition using mirror visual feedback improved by transcranial direct current stimulation (tDCS) in patients with Parkinson's disease," Frontiers in Neuroscience, vol. 13, p. 602, 2019.

[23] F. Fregni, P. S. Boggio, M. A. Nitsche, S. P. Rigonatti, and A. Pascual-Leone, "Cognitive effects of repeated sessions of transcranial direct current stimulation in patients with depression," Depression and Anxiety, vol. 23, no. 8, pp. 482484, 2006.

[24] F. Fregni, P. Boggio, C. Mansur et al., "Transcranial direct current stimulation of the unaffected hemisphere in stroke patients," Neuroreport, vol. 16, no. 14, pp. 1551-1555, 2005. 
[25] F. Hummel, P. Celnik, P. Giraux et al., "Effects of noninvasive cortical stimulation on skilled motor function in chronic stroke," Brain, vol. 128, no. 3, pp. 490-499, 2005.

[26] M. C. W. English, E. S. Kitching, M. T. Maybery, and T. A. W. Visser, "Modulating attentional biases of adults with autistic traits using transcranial direct current stimulation: a pilot study," Autism Research, vol. 11, no. 2, pp. 385-390, 2018.

[27] J. Kang, E. Cai, J. Han et al., "Transcranial direct current stimulation (tDCS) can modulate EEG complexity of children with autism spectrum disorder," Frontiers in Neuroscience, vol. 12, p. 201, 2018.

[28] L. Marshall, M. Mölle, M. Hallschmid, and J. Born, "Transcranial direct current stimulation during sleep improves declarative memory," Journal of Neuroscience, vol. 24, no. 44, pp. 9985-9992, 2004.

[29] J. C. de Souza Custódio, C. W. Martins, M. D. M. V. Lugon, F. Fregni, and E. M. Nakamura-Palacios, "Epidural direct current stimulation over the left medial prefrontal cortex facilitates spatial working memory performance in rats," Brain Stimulation, vol. 6, no. 3, pp. 261-269, 2013.

[30] X. Yu, Y. Li, H. Wen, Y. Zhang, and X. Tian, "Intensitydependent effects of repetitive anodal transcranial direct current stimulation on learning and memory in a rat model of Alzheimer's disease," Neurobiology of Learning and Memory, vol. 123, pp. 168-178, 2015.

[31] K. J. Yoon, Y. T. Lee, S. W. Chae, C. R. Park, and D. Y. Kim, "Effects of anodal transcranial direct current stimulation (tDCS) on behavioral and spatial memory during the early stage of traumatic brain injury in the rats," Journal of the Neurological Sciences, vol. 362, pp. 314-320, 2016.

[32] T. Kamida, S. Kong, N. Eshima, T. Abe, M. Fujiki, and H. Kobayashi, "Transcranial direct current stimulation decreases convulsions and spatial memory deficits following pilocarpine-induced status epilepticus in immature rats," Behavioural Brain Research, vol. 217, no. 1, pp. 99-103, 2011.

[33] D. T. Leffa, A. de Souza, V. L. Scarabelot et al., “Transcranial direct current stimulation improves short-term memory in an animal model of attention-deficit/hyperactivity disorder," European Neuropsychopharmacology, vol. 26, no. 2, pp. 368377, 2016.

[34] D. Moher, A. Liberati, J. Tetzlaff, D. G. Altman, and The PRISMA Group, "Preferred reporting items for systematic reviews and meta-analyses: the PRISMA statement," PLoS Medicine, vol. 6, no. 7, article e1000097, 2009.

[35] M. R. Krause, T. P. Zanos, B. A. Csorba et al., “Transcranial direct current stimulation facilitates associative learning and alters functional connectivity in the primate brain," Current Biology, vol. 27, no. 20, pp. 3086-3096.e3, 2017.

[36] F. Manteghi, M. Nasehi, and M. Zarrindast, "Precondition of right frontal region with anodal tDCS can restore the fear memory impairment induced by ACPA in male mice," EXCLI Journal, vol. 16, pp. 1-13, 2017.

[37] M. Nasehi, R. Soltanpour, M. Ebrahimi-Ghiri, S. Zarrabian, and M. R. Zarrindast, "Interference effects of transcranial direct current stimulation over the right frontal cortex and adrenergic system on conditioned fear," Psychopharmacology, vol. 234, no. 22, pp. 3407-3416, 2017.

[38] M. Nasehi, M. Khani-Abyaneh, M. Ebrahimi-Ghiri, and M. R. Zarrindast, "The effect of left frontal transcranial direct-current stimulation on propranolol-induced fear memory acquisition and consolidation deficits," Behavioural Brain Research, vol. 331, pp. 76-83, 2017.
[39] S. Abbasi, M. Nasehi, H. R. S. Lichaei, and M. R. Zarrindast, "Effects of left prefrontal transcranial direct current stimulation on the acquisition of contextual and cued fear memory," Iranian Journal of Basic Medical Sciences, vol. 20, no. 6, pp. 623-630, 2017.

[40] M. Podda, S. Cocco, A. Mastrodonato et al., "Anodal transcranial direct current stimulation boosts synaptic plasticity and memory in mice via epigenetic regulation of Bdnf expression," Scientific Reports, vol. 6, p. 22180, 2016.

[41] T. H. Yu, Y. J. Wu, M. E. Chien, and K. S. Hsu, "Transcranial direct current stimulation induces hippocampal metaplasticity mediated by brain-derived neurotrophic factor," Neuropharmacology, vol. 144, no. 1, pp. 358-367, 2019.

[42] C. Dockery, D. Liebetanz, N. Birbaumer, M. Malinowska, and M. J. Wesierska, "Cumulative benefits of frontal transcranial direct current stimulation on visuospatial working memory training and skill learning in rats," Neurobiology of Learning and Memory, vol. 96, no. 3, pp. 452-460, 2011.

[43] S. Yu, S. Park, and K. Sim, "The effect of tDCS on cognition and neurologic recovery of rats with Alzheimer's disease," Journal of Physical Therapy Science, vol. 26, no. 2, pp. 247249, 2014.

[44] C. H. Chang, H. Y. Lane, and C. H. Lin, "Brain stimulation in Alzheimer's disease," Frontiers in Psychiatry, vol. 9, 2018.

[45] A. Roostaei, G. Vaezi, M. Nasehi, A. Haeri-Rohani, and M. R. Zarrindast, "The Involvement of D1 and D2 dopamine receptors in the restoration effect of left frontal anodal, but not cathodal, tDCS on streptozocin-induced amnesia," Archives of Iranian Medicine, vol. 22, no. 3, pp. 144-154, 2019.

[46] Y. Wu, C. Lin, C. Yeh et al., "Repeated transcranial direct current stimulation improves cognitive dysfunction and synaptic plasticity deficit in the prefrontal cortex of streptozotocininduced diabetic rats," Brain Stimulation, vol. 10, no. 6, pp. 1079-1087, 2017.

[47] D. T. Leffa, B. Bellaver, A. A. Salvi et al., "Transcranial direct current stimulation improves long-term memory deficits in an animal model of attention-deficit/hyperactivity disorder and modulates oxidative and inflammatory parameters," Brain Stimulation, vol. 11, no. 4, pp. 743-751, 2018.

[48] O. Bragina, D. Lara, E. Nemoto, C. W. Shuttleworth, O. V. Semyachkina-Glushkovskaya, and D. E. Bragin, "Increases in microvascular perfusion and tissue oxygenation via vasodilatation after anodal transcranial direct current stimulation in the healthy and traumatized mouse brain," Advances in Experimental Medicine and Biology, vol. 1072, pp. 27-31, 2018.

[49] P. Spagnolo and D. Goldman, "Neuromodulation interventions for addictive disorders: challenges, promise and roadmap for future research," Brain, vol. 140, no. 5, pp. 11831203, 2017.

[50] S. Pedron, J. Monnin, E. Haffen, D. Sechter, and V. van Waes, "Repeated transcranial direct current stimulation prevents abnormal behaviors associated with abstinence from chronic nicotine consumption," Neuropsychopharmacology, vol. 39, no. 4, pp. 981-988, 2014.

[51] S. Pedron, J. Beverley, E. Haffen, P. Andrieu, H. Steiner, and V. van Waes, "Transcranial direct current stimulation produces long-lasting attenuation of cocaine-induced behavioral responses and gene regulation in corticostriatal circuits," Addiction Biology, vol. 22, no. 5, pp. 1267-1278, 2017.

[52] T. Kamida, S. Kong, N. Eshima, and M. Fujiki, "Cathodal transcranial direct current stimulation affects seizures and 
cognition in fully amygdala- kindled rats," Neurological Research, vol. 35, no. 6, pp. 602-607, 2013.

[53] M. Zobeiri and G. van Luijtelaar, "Noninvasive transcranial direct current stimulation in a genetic absence model," Epilepsy \& Behavior, vol. 26, no. 1, pp. 42-50, 2013.

[54] S. C. Dhamne, D. Ekstein, Z. Zhuo et al., "Acute seizure suppression by transcranial direct current stimulation in rats," Annals of Clinical and Translational Neurology, vol. 2, no. 8, pp. 843-856, 2015.

[55] J. G. Rohan, K. A. Carhuatanta, S. M. McInturf, M. K. Miklasevich, and R. Jankord, "Modulating hippocampal plasticity with in vivo brain stimulation," The Journal of Neuroscience, vol. 35, no. 37, pp. 12824-12832, 2015.

[56] E. Gondard, M. Soto-Montenegro, A. Cassol, A. M. Lozano, and C. Hamani, "Transcranial direct current stimulation does not improve memory deficits or alter pathological hallmarks in a rodent model of Alzheimer's disease," Journal of Psychiatric Research, vol. 114, pp. 93-98, 2019.

[57] K. J. Yoon, B. M. Oh, and D. Y. Kim, "Functional improvement and neuroplastic effects of anodal transcranial direct current stimulation (tDCS) delivered 1 day vs. 1 week after cerebral ischemia in rats," Brain Research, vol. 1452, pp. 61-72, 2012.

[58] D. Hebb, The Organization of Behavior, Wiley, New York, 1949.

[59] M. Rueger, M. Keuters, M. Walberer et al., "Multi-session transcranial direct current stimulation (tDCS) elicits inflammatory and regenerative processes in the rat brain," PLoS One, vol. 7, no. 8, article e43776, 2012.

[60] L. Peruzzotti-Jametti, M. Cambiaghi, M. Bacigaluppi et al., "Safety and efficacy of transcranial direct current stimulation in acute experimental ischemic stroke," Stroke, vol. 44, no. 11, pp. 3166-3174, 2013.

[61] C. McCaig, L. Sangster, and R. Stewart, "Neurotrophins enhance electric field-directed growth cone guidance and directed nerve branching," Developmental Dynamics, vol. 217, no. 3, pp. 299-308, 2000.

[62] A. Pikhovych, N. P. Stolberg, L. Jessica Flitsch et al., “Transcranial direct current stimulation modulates neurogenesis and microglia activation in the mouse brain," Stem Cells International, vol. 2016, 9 pages, 2016.

[63] J. Márquez-Ruiz, R. Leal-Campanario, R. SánchezCampusano et al., "Transcranial direct-current stimulation modulates synaptic mechanisms involved in associative learning in behaving rabbits," Proceedings of the National Academy of Sciences of the United States of America, vol. 109, no. 17, pp. 6710-6715, 2012.

[64] B. Krause, J. Márquez-Ruiz, and R. C. Kadosh, "The effect of transcranial direct current stimulation: a role for cortical excitation/inhibition balance?," Frontiers in Human Neuroscience, vol. 7, no. 602, 2013.

[65] D. Antonenko, F. Schubert, F. Bohm et al., "tDCS-induced modulation of GABA levels and resting-state functional connectivity in older adults," The Journal of Neuroscience, vol. 37, no. 15, pp. 4065-4073, 2017.

[66] C. Stagg and M. Nitsche, "Physiological basis of transcranial direct current stimulation," The Neuroscientist, vol. 17, no. 1, pp. 37-53, 2011.

[67] C. J. Stagg, V. Bachtiar, U. Amadi et al., "Local GABA concentration is related to network-level resting functional connectivity," eLife, vol. 3, no. 3, pp. 1-9, 2014.
[68] B. Fritsch, J. Reis, K. Martinowich et al., "Direct current stimulation promotes BDNF-dependent synaptic plasticity: potential implications for motor learning," Neuron, vol. 66, no. 2, pp. 198-204, 2010.

[69] F. Ranieri, M. Podda, E. Riccardi et al., "Modulation of LTP at rat hippocampal CA3-CA1 synapses by direct current stimulation," Journal of Neurophysiology, vol. 107, no. 7, pp. 18681880, 2012.

[70] G. Kronberg, M. Bridi, T. Abel, M. Bikson, and L. C. Parra, "Direct current stimulation modulates LTP and LTD: activity dependence and dendritic effects," Brain Stimulation, vol. 10, no. 1, pp. 51-58, 2017.

[71] Y. Sun, J. Lipton, L. Boyle et al., "Direct current stimulation induces mGluR5-dependent neocortical plasticity," Annals of Neurology, vol. 80, no. 2, pp. 233-246, 2016.

[72] C. Lüscher and R. Malenka, "NMDA receptor-dependent long-term potentiation and long-term depression (LTP/LTD)," Cold Spring Harbor Perspectives in Biology, vol. 4, no. 6, p. a005710, 2012.

[73] J. Stafford, M. Brownlow, A. Qualley, and R. Jankord, "AMPA receptor translocation and phosphorylation are induced by transcranial direct current stimulation in rats," Neurobiology of Learning and Memory, vol. 150, pp. 36-41, 2018.

[74] S. Perret, A. Cantereau, J. Audin, B. Dufy, and D. Georgescauld, "Interplay between $\mathrm{Ca}^{2+}$ release and $\mathrm{Ca}^{2+}$ influx underlies localized hyperpolarization-induced $\left[\mathrm{Ca}^{2+}\right] \mathrm{i}$ waves in prostatic cells," Cell Calcium, vol. 25, no. 4, pp. 297-311, 1999.

[75] H. Monai, M. Ohkura, M. Tanaka et al., "Calcium imaging reveals glial involvement in transcranial direct current stimulation-induced plasticity in mouse brain," Nature Communications, vol. 7, p. 11100, 2016.

[76] T. Mishima, T. Nagai, K. Yahagi et al., "Transcranial direct current stimulation (tDCS) induces adrenergic receptordependent microglial morphological changes in mice," eNeuro, vol. 6, no. 5, pp. 1-12, 2019.

[77] P. Opazo, S. Labrecque, C. Tigaret et al., "CaMKII triggers the diffusional trapping of surface AMPARs through phosphorylation of stargazin," Neuron, vol. 67, no. 2, pp. 239-252, 2010.

[78] M. S. Kim, H. Koo, S. W. Han et al., "Repeated anodal transcranial direct current stimulation induces neural plasticity-associated gene expression in the rat cortex and hippocampus," Restorative Neurology and Neuroscience, vol. 35, no. 2, pp. 137-146, 2017.

[79] G. Leal, C. Bramham, and C. Duarte, "BDNF and hippocampal synaptic plasticity," Vitamins and Hormones, vol. 104, pp. 153-195, 2017.

[80] C. McCaig, A. Rajnicek, B. Song, and M. Zhao, "Controlling cell behavior electrically: current views and future potential," Physiological Reviews, vol. 85, no. 3, pp. 943-978, 2005.

[81] L. Minichiello, "TrkB signalling pathways in LTP and learning," Nature Reviews Neuroscience, vol. 10, no. 12, pp. 850860, 2009.

[82] C. M. Alberini, "Transcription factors in long-term memory and synaptic plasticity," Physiological Reviews, vol. 89, no. 1, pp. 121-145, 2009.

[83] S. T. Carmichael, I. Archibeque, L. Luke, T. Nolan, J. Momiy, and $\mathrm{S}$. Li, "Growth-associated gene expression after stroke: evidence for a growth- promoting region in peri-infarct 
cortex," Experimental Neurology, vol. 193, no. 2, pp. 291-311, 2005.

[84] Y. Li, N. Jiang, C. Powers, and M. Chopp, "Neuronal damage and plasticity identified by microtubule-associated protein 2 , growth-associated protein 43 , and cyclin D1 immunoreactivity after focal cerebral ischemia in rats," Stroke, vol. 29, no. 9, pp. 1972-1981, 1998.

[85] T. Jiang, R. X. Xu, A. W. Zhang et al., "Effects of transcranial direct current stimulation on hemichannel pannexin-1 and neural plasticity in rat model of cerebral infarction," Neurosciences, vol. 226, pp. 421-426, 2012.

[86] P. Bargiotas, H. Monyer, and M. Schwaninger, "Hemichannels in cerebral ischemia," Current Molecular Medicine, vol. 9, no. 2, pp. 186-194, 2009.

[87] L. Dimov, A. Franciosi, A. Campos, A. R. Brunoni, and R. L. Pagano, "Top-down effect of direct current stimulation on the nociceptive response of rats," PLoS One, vol. 11, no. 4, article e0153506, 2016.

[88] M. Rioult-Pedotti, D. Friedman, and J. Donoghue, "Learninginduced LTP in neocortex," Science, vol. 290, no. 5491, pp. 533-536, 2000.

[89] R. Malenka and M. Bear, "LTP and LTD: an embarrassment of riches," Neuron, vol. 44, no. 1, pp. 5-21, 2004.

[90] J. Lisman, K. Cooper, M. Sehgal, and A. J. Silva, "Memory formation depends on both synapse-specific modifications of synaptic strength and cell-specific increases in excitability," Nature Neuroscience, vol. 21, no. 3, pp. 309-314, 2018.

[91] M. Legrand, R. Troubat, B. Brizard, A. M. le Guisquet, C. Belzung, and W. el-Hage, "Prefrontal cortex rTMS reverses behavioral impairments and differentially activates c-Fos in a mouse model of post-traumatic stress disorder," Brain Stimulation, vol. 12, no. 1, pp. 87-95, 2019.

[92] V. Moliadze, E. Lyzhko, T. Schmanke, S. Andreas, C. M. Freitag, and M. Siniatchkin, "1 mA cathodal tDCS shows excitatory effects in children and adolescents: insights from TMS evoked N100 potential," Brain Research Bulletin, vol. 140, pp. 43-51, 2018.

[93] F. Fisicaro, G. Lanza, A. A. Grasso et al., "Repetitive transcranial magnetic stimulation in stroke rehabilitation: review of the current evidence and pitfalls," Therapeutic Advances in Neurological Disorders, vol. 12, p. 1756286419878317, 2019.

[94] M. Cantone, A. Bramanti, G. Lanza et al., "Cortical plasticity in depression," ASN Neuro, vol. 9, no. 3, 2017.

[95] U. Palm, F. M. Segmiller, A. N. Epple et al., "Transcranial direct current stimulation in children and adolescents: a comprehensive review," Journal of Neural Transmission (Vienna), vol. 123, no. 10, pp. 1219-1234, 2016.

[96] X. Leinekugel, I. Khalilov, H. McLean et al., "GABA is the principal fast-acting excitatory transmitter in the neonatal brain," Advances in Neurology, vol. 79, pp. 189-201, 1999.

[97] C. W. Martins, L. C. de Melo Rodrigues, M. A. Nitsche, and E. M. Nakamura-Palacios, "AMPA receptors are involved in prefrontal direct current stimulation effects on long-term working memory and GAP-43 expression," Behavioural Brain Research, vol. 362, pp. 208-212, 2019.

[98] V. Nekhendzy, C. Fender, M. Davies et al., "The antinociceptive effect of transcranial electrostimulation with combined direct and alternating current in freely moving rats," Anesthesia and Analgesia, vol. 98, no. 3, pp. 730-7, table of contents, 2004.
[99] N. O. B. Taib and M. Manto, "Trains of transcranial direct current stimulation antagonize motor cortex hypoexcitability induced by acute hemicerebellectomy," Journal of Neurosurgery, vol. 111, no. 4, pp. 796-806, 2009.

[100] S. Kim, B. Kim, Y. Ko, M. S. Bang, M. H. Kim, and T. R. Han, "Functional and histologic changes after repeated transcranial direct current stimulation in rat stroke model," Journal of Korean Medical Science, vol. 25, no. 10, pp. 1499-1505, 2010.

[101] G. Laste, W. Caumo, L. Adachi et al., "After-effects of consecutive sessions of transcranial direct current stimulation (tDCS) in a rat model of chronic inflammation," Experimental Brain Research, vol. 221, no. 1, pp. 75-83, 2012.

[102] L. N. S. Adachi, W. Caumo, G. Laste et al., "Reversal of chronic stress-induced pain by transcranial direct current stimulation (tDCS) in an animal model," Brain Research, vol. 1489, pp. 17-26, 2012.

[103] F. Notturno, M. Pace, F. Zappasodi, E. Cam, C. L. Bassetti, and A. Uncini, "Neuroprotective effect of cathodal transcranial direct current stimulation in a rat stroke model," Journal of the Neurological Sciences, vol. 342, no. 1-2, pp. 146-151, 2014.

[104] C. Lu, Y. Wei, R. Hu, Y. Wang, K. Li, and X. Li, “Transcranial Direct Current Stimulation Ameliorates Behavioral Deficits and Reduces Oxidative Stress in 1-Methyl-4-Phenyl-1,2,3,6Tetrahydropyridine-Induced Mouse Model of Parkinson's Disease," Neuromodulation, vol. 18, no. 6, pp. 442-447, 2015.

[105] L. N. S. Adachi, A. S. Quevedo, A. de Souza et al., "Exogenously induced brain activation regulates neuronal activity by top-down modulation: conceptualized model for electrical brain stimulation," Experimental Brain Research, vol. 233, no. 5, pp. 1377-1389, 2015.

[106] Y.-H. Liu, L.-D. Liao, S. J. Chan, A. Bandla, and N. V. Thakor, "An integrated neuroprotective intervention for brain ischemia validated by ECoG- fPAM," in 2016 38th Annual International Conference of the IEEE Engineering in Medicine and Biology Society (EMBC), pp. 4009-4012, Orlando, FL, USA, Aug. 2016.

[107] R. Braun, R. Klein, H. Walter et al., "Transcranial direct current stimulation accelerates recovery of function, induces neurogenesis and recruits oligodendrocyte precursors in a rat model of stroke," Experimental Neurology, vol. 279, pp. 127-136, 2016.

[108] S. G. Cioato, L. F. Medeiros, P. R. M. Filho et al., "Long-lasting effect of transcranial direct current stimulation in the reversal of hyperalgesia and cytokine alterations induced by the neuropathic pain model," Brain Stimulation, vol. 9, no. 2, pp. 209-217, 2016.

[109] P. R. M. Filho, R. Vercelino, S. G. Cioato et al., "Transcranial direct current stimulation (tDCS) reverts behavioral alterations and brainstem BDNF level increase induced by neuropathic pain model: Long- lasting effect," Progress in Neuro-Psychopharmacology \& Biological Psychiatry, vol. 64, pp. 44-51, 2016.

[110] S. F. da Silva Moreira, L. F. Medeiros, A. de Souza et al., "Transcranial direct current stimulation (tDCS) neuromodulatory effects on mechanical hyperalgesia and cortical BDNF levels in ovariectomized rats," Life Sciences, vol. 145, pp. 233-239, 2016.

[111] Y. Liu, S. Chan, H. Pan et al., "Integrated treatment modality of cathodal-transcranial direct current stimulation with 
peripheral sensory stimulation affords neuroprotection in a rat stroke model," Neurophotonics, vol. 4, no. 4, article 045002, 2017.

[112] C. Winkler, J. Reis, N. Hoffmann et al., "Anodal transcranial direct current stimulation enhances survival and integration of dopaminergic cell transplants in a rat Parkinson model," eNeuro, vol. 4, no. 5, pp. 1-11, 2017.

[113] A. Souza, D. F. Martins, L. F. Medeiros et al., "Neurobiological mechanisms of antiallodynic effect of transcranial direct current stimulation (tDCS) in a mice model of neuropathic pain," Brain Research, vol. 1682, pp. 14-23, 2018.

[114] F. Paciello, M. V. Podda, R. Rolesi et al., "Anodal transcranial direct current stimulation affects auditory cortex plasticity in normal-hearing and noise-exposed rats," Brain Stimulation, vol. 11, no. 5, pp. 1008-1023, 2018.

[115] F. Fregni, I. Macedo, L. Spezia-Adachi et al., "Transcranial direct current stimulation (tDCS) prevents chronic stressinduced hyperalgesia in rats," Brain Stimulation, vol. 11, no. 2, pp. 299-301, 2018.

[116] S. Lee, J. Youn, W. Jang, and H. O. Yang, "Neuroprotective effect of anodal transcranial direct current stimulation on 1-methyl-4-phenyl-1,2,3,6-tetrahydropyridine (MPTP)induced neurotoxicity in mice through modulating mitochondrial dynamics," Neurochemistry International, vol. 129, p. 104491, 2019.

[117] E. M. M. Callai, V. L. Scarabelot, L. Fernandes Medeiros et al., "Transcranial direct current stimulation (tDCS) and trigeminal pain: a preclinical study," Oral Diseases, vol. 25, no. 3, pp. 888-897, 2019.

[118] V. Scarabelot, C. de Oliveira, L. Medeiros et al., "Transcranial direct-current stimulation reduces nociceptive behaviour in an orofacial pain model," Journal of Oral Rehabilitation, vol. 46, no. 1, pp. 40-50, 2019. 